\title{
Observations of the global haze redistribution on Titan from 2006 to 2015 with OSIRIS at Keck
}

\author{
Máté Ádámkovics and Imke de Pater \\ ${ }^{a}$ Astronomy Department, University of California, Berkeley, CA 94720-3411
}

\begin{abstract}
We observed Titan with the OH Suppressing InfraRed Imaging Spectrograph (OSIRIS) at the W. M. Keck observatory from 2006 through 2015 using adaptive optics. The sunlight scattered by atmospheric haze was spatially resolved in the $2.0 \mu \mathrm{m}(\mathrm{K})$ band window, and the spectra were analyzed with a radiative transfer model to determine the vertical (altitude) and meridional (latitudinal) variation in the haze distribution over this time period. This study complements recent work by Karkoschka (2016) in the season of observations, in the time span and sampling interval, in wavelength coverage and spectral resolution, as well as in the radiative transfer methodology and analysis. We observe the largest meridional gradient in haze opacity above $20 \mathrm{~km}$ toward the northern hemisphere in January 2010. Individual observations can show significant deviations from a relatively smooth linear gradient in haze across the entire disk. The variation in haze below $20 \mathrm{~km}$ is rarely well-described by a simple model and there is a systematically smaller amount of haze opacity retrieved from the Equator to $10^{\circ} \mathrm{S}$ when observing the disk with a sub-observer longitude near $150^{\circ} \mathrm{W}$. This correlation with longitude suggests one of the following; a localized decrease in haze scattering, a localized increase in gas opacity, or a systematic over-estimate of the surface albedo in this region.
\end{abstract}

Keywords: Titan, atmosphere, Adaptive optics, Atmospheres, evolution, Atmospheres, structure

Email address: mate@berkeley.edu (Máté Ádámkovics)

Preprint submitted to Icarus

February 13, 2017

(C) 2016. This manuscript version is made available under the Elsevier user license http://www.elsevier.com/open-access/userlicense/1.0/ 


\section{Introduction}

The hydrocarbon hazes on Titan are one tracer of the overturning circulation (e.g., Smith et al., 1981; Lorenz et al., 2001; Rannou et al., 2004; Larson et al., 2015). Together with the location and frequency of cloud formation, the distribution and evolution of hazes provide insight into the photochemistry and dynamics of the atmosphere (e.g., Strobel, 1974; Yung et al., 1984; Wilson and Atreya, 2004; Lavvas et al., 2008b; Krasnopolsky, 2009; Willacy et al., 2016). Podolak et al. (1979) noted the general acceptance of so-called "Axel dust" in the upper atmosphere of Titan as they followed the early laboratory experiments of Khare and Sagan (1973) in trying to understand the optical and chemical properties of this material. Allen et al. (1980) proposed a photochemical theory of the production of the polymeric compounds. Early models of the solar phase angle variations of Titan's geometric albedo (Rages and Pollack, 1980) were used to determine the optical properties and vertical distribution of this material on a global scale. Voyager observations would provide the first resolved look at the hazes on Titan (Smith et al., 1981, 1982).

The properties of the sunlight that is scattered by hazes in the atmosphere is dependent on the shape and size distribution of particulates (Rages et al., 1983; McKay et al., 1989; Tomasko et al., 2008; Doose et al., 2016; Mishchenko et al., 2016), which vary spatially with geographic location as well as vertically, in altitude above the surface (e.g., Rages and Pollack, 1983; Lorenz et al., 2001; Anderson et al., 2008). Toon et al. (1992) presented a model to explain the hemispheric asymetry that was observed Voyager as a result of the Hadley-type motions above $300 \mathrm{~km}$. These models were used to simulate the time-dependent production of haze, in order to reconcile the predicted geometric albedo of Titan with observations (Hutzell et al., 1993), before they were extended to two dimensions in order to interpret brightness variations observed with both season and latitude (Hutzell et al., 1996) The state of knowledge about aerosols on Titan, prior to the arrival of Cassini, was reviewed by McKay et al. (2001). Recent microphysical models of the vertical distribution of haze (Lavvas et al., 2008a) build on treat the photochemical processes that turn atmospheric methane gas into the larger hydrocarbon molecules as well as the details of the condensation process through heterogeneous nucleation and subsequent coagulation. The haze distributions in these models are solved self-consistently with the atmospheric thermal structure and are in agreement with the properties measured by the Descent 
Imager/Spectral Radiometer (DISR) on the Huygens probe (Tomasko et al., 2005, 2008; Lavvas et al., 2011). While the measurements made by instruments on Cassini and Huygens provide the most detailed constraints for our understanding of the vertical structure of haze, the hazes on Titan are neither uniformly distributed nor static. The role of the seasonally changing insolation, and the resulting impact on the photochemistry that is related to haze formation, is not well constrained against the role of transport by large scale dynamics (e.g., Rannou et al., 2002), suggesting that additional information about the temporal evolution of hazes is valuable for evaluating the photochemistry and dynamics on Titan.

The Hubble Space Telescope (HST) was used to measure latitudinal variation in the size of aerosol (Karkoschka and Lorenz, 1997), and subsequent observations with HST revealed a stronger hemispheric contrast than expected (Lorenz et al., 1999) with changes in the brightness distribution over seasonal timescales and large scale albedo variations near the pole (Lorenz et al., 2001, 2004). A 3-dimensional map the haze in the lower atmosphere (in 1996), from $\sim 100 \mathrm{~km}$ to the surface was assembled using $6 H S T$ filters in the 888 to $953 \mathrm{~nm}$ range (Young et al., 2002), showing an upper atmospheric aerosol (above $16 \mathrm{~km}$ ) that peaked near the equator and fell off toward both poles, and a lower atmospheric haze (below $16 \mathrm{~km}$ ) that shows peak optical depths at both northern and southern mid-latitudes (near 30-40 ).

These haze asymetries can also be observed from telescopes on Earth in the near-IR (e.g., Gibbard et al., 1999; Roe et al., 2002; Ádámkovics et al., 2004, 2006). The Visual and Infrared Mapping Spectrometer (VIMS) on Cassini (Brown et al., 2004) has been used to measure the distribution of haze from 2004 through 2008, finding both gradual gradients as well as stepwise discontinuities in the haze (e.g., Rannou et al., 2010; Penteado et al., 2010). Spatially discrete features such as polar haze enhancements have also been observed with VIMS (Rannou et al., 2012), while a tropical haze band was observed using the Imaging Science Subsystem (ISS), together with VIMS and the Cassini Composite InfraRed Spectrometer (CIRS) (de Kok et al., 2010).

The high-altitude detached haze layers were initially observed by the Cassini ISS to be significantly higher (above $500 \mathrm{~km}$ ) than measured by Voyager (below $400 \mathrm{~km}$; Rages and Pollack, 1983). Near equinox, dramatic changes in vertical structure of the hazes were observed, with the altitude of the upper detached haze layer dropping from over $500 \mathrm{~km}$ down to below $380 \mathrm{~km}$ between 2007 and 2010, thereby connecting the Cassini observations 
with Voyager measurements from the previous seasonal cycle (West et al., 2011). The evolution of the detached photochemical haze layer is one observational constraint in evaluating our understanding the relative importance of the haze microphysics, as regulated by variations in the photochemistry that are driven by insolation, compared to the role of global dynamics, wave structures, and winds. Meier et al. (2000) used Hubble Space Telescope (HST) observations in 1997 and 1998 to tentatively identify banded (zonal) haze structures close to the surface. Starting from the same epoch, Gibbard et al. (2004) measured the $2 \mu \mathrm{m}$ haze opacity using speckle and adaptive optics imaging at the W. M. Keck Observatory, finding the Southern haze opacity to be decreasing by a factor of about 2 from 1996 through 2004, with equatorial hazes staying relatively constant. Recently, Karkoschka (2016) presented an analysis of HST Space Telescope Imaging Spectrograph (STIS) observations of Titan from five years between 1997 and 2004. Those observations at 543 and $990 \mathrm{~nm}$ wavelengths were use to show that there were two separate seasonally varying components of atmospheric haze opacity above and below $\sim 100 \mathrm{~km}$ altitude. The extrema for the observed variation in haze opacities occurred in the earliest measurements in 1997, and there was a 2 year time period from 2002 through 2004 where the hemispheric asymmetries reversed in low, mid, and high latitudes.

Constraining the spatial variation in hazes closest to the surface, using wavelength windows that are most sensitive to the surface, is important for determining atmospheric properties in the boundary layer. Spatial variation in boundary layer methane is related to the source region(s) of methane (Ádámkovics et al., 2016), however, degeneracies between methane absorption and haze scattering in retrieval algorithms complicate an unambiguous determination of the methane abundance. Placing constraints on the low altitude haze opacity can therefore inform the retrievals of the methane abundance in the boundary layer. Here we present observations of haze variation that are complementary to a recent study by Karkoschka (2016). Our 14 observations, from early 2006 through mid 2015, are presented in Section 2. As well as covering a different season on Titan, our observations are in a longer wavelength band at $2 \mu \mathrm{m}$, as well as at higher spectral resolution. These near-infrared, ground-based observations of the haze evolution on Titan are interpreted with a radiative transfer model, discussed in Section 3. We present our results of fitting the haze variations in Section 4 and discuss the results in Section 5. 


\section{Observations}

Titan was observed from January 2006 through July 2015 with the OH Suppressing InfraRed Imaging Spectrograph (OSIRIS) at the W. M. Keck Observatory. OSIRIS is a near-IR integral-field spectrograph (Larkin et al., 2006) that operates in the $z, J, H$ and $K$ bands, with a nominal spectral resolution of $\lambda / \Delta \lambda=3700$ (roughly $5 \AA$ ) and a diffraction-limited spatial resolution approaching 60 mas in $\mathrm{K}$ band provided by the adaptive optics system (Wizinowich et al., 2000). The instrument was mounted to the Nasmyth platform of the Keck II telescope over the timespan of this observing campaign and was moved to Keck I in 2012.

For most of the campaign we used the broadband Kbb $(1.965-2.381 \mu \mathrm{m})$ filters at a 20 mas plate scale, which corresponds to a $0.32 \times 1.28$ " field of view (FOV) with 1665 spectral channels. The observed diameter of Titan when including the atmosphere is $\sim 1 "$, so 4 exposures were spatially mosaicked for full disk coverage. The OSIRIS reduction pipeline was used for the following standard processing steps: flat-fielding, sky subtraction, cosmic ray removal, channel level adjustment and the removal of crosstalk, before the extraction of spectra and assembly of a datacube. The reduction pipeline was recently described as part of the work by Iserlohe et al. (2013). Mosaicking via the OSIRIS pipeline was unreliable and was instead performed manually. Datacubes were collapsed over wavelength to create an image. The exposures near the center of Titan that sampled both the North and South limbs were used to fix the apparent diameter and a circle was drawn. The exposures near the East and West limbs were then offset to within a 20 mas pixel in order to produce a circular image of the disk. A gradual mechanical deterioration of the lenslet mask caused the leakage of light across channels and was manifest as artificially bright pixels at the edge of the FOV. These pixels were masked as necessary, however, since the magnitude of this artifact varies with wavelength, it was not possible to completely mask out all edge pixels without also excluding valid data. Therefore, artifacts at the edges of some images remain, manifested as bright vertical stripes in the mosaicked datacubes. To a lesser degree there are also faint vertical stripes near the center of the disk in some images. Later in the observing campaign we switched to narrow-band Kn2 $(2.036-2.141 \mu \mathrm{m})$ and Kn3 $(2.121-2.229 \mu \mathrm{m})$ filters, which at a 20 mas plate scale offer $0.90 \times 1.28$ " FOV that covers nearly the entire disk of Titan and eliminates the need for spatial mosaicking. The Kn2 and Kn3 filters combine to have 854 spectral channels with slightly abridged 
wavelength coverage relative to the Kbb filter. Depending on the angular diameter of Titan, and possible vignetting, we recorded $\sim 1100-1500$ spatial pixels on the disk, resulting in cubes of $\sim 2 \times 10^{6}$ datapoints in the Kbb filter and half that in the combination of $\mathrm{Kn} 2$ and Kn3 filters, for a total of $2.62 \times 10^{7}$ spatial-spectral datapoints in all of the observations.

On each night an A-type star with $J, H$, and $K$ magnitudes from $2 M A S S$ was used to correct for telluric absorption, instrument spectral response, and for photometry as described by Laver et al. (2007). A second-order photometric correction was performed using 113 IRTF spectra (Brown, M. E., private communication). The light curves for Titan at wavelengths sensitive to the surface $(2.017-2.044 \mu \mathrm{m})$ and stratosphere $(2.216-2.266 \mu \mathrm{m})$ were fit to the calibrated IRTF measurements and used to provide an offset and scale factor correction for the disk-integrated OSIRIS observations. The log of the observations is presented in Table 1, and disk-integrated spectra that characterize the datacubes are presented in Figure 1.

\section{Radiative transfer model}

Synthetic spectra are generated by defining 20 atmospheric layers, with properties that are determined primarily by measurements made with instruments on the Huygens probe. The layers have boundaries (levels) that are evenly spaced in pressure, with 10 levels above and 10 levels below 300 mbar (see Table 2 in Ádámkovics et al., 2016). The top of the atmosphere is set at zero optical depth. We use measurements of the temperature, pressure, methane abundance, and haze structure to determine the gas and scattering opacity in each layer. The Huygens temperature profile is used at all latitudes. $\mathrm{CH}_{4}$ and $\mathrm{CH}_{3} \mathrm{D}$ line opacities are from the HITRAN 2012 database (Rothman et al., 2013). The discrete-ordinate-method radiative transfer(DISORT; Stamnes et al., 1988) is implemented in Python (PyDISORT) and used to solve the radiative transfer through the model atmosphere and simulate the observed flux (Ádámkovics et al., 2016). We use the pseudospherical correction to plane-parallel geometry in DISORT to account for Titan's thick atmosphere and limit our analysis to viewing geometries on Titan with solar zenith angles below $50^{\circ}$, where the correction is smaller than other sources of uncertainty.

Each $(x, y)$ pixel in a datacube maps to a spectrum at a given latitude and longitude, with a corresponding incidence angle $\theta_{0}$, and emission angle, $\theta_{e}$. Below we use the standard notation, $\mu_{0} \equiv \cos \theta_{0}$ and $\mu_{e} \equiv \cos \theta_{e}$. Since $\mu_{0} \approx$ 
Table 1: Log of Observations

\begin{tabular}{ccccrrrrr}
\hline Date (UTC) & Filter & ${ }^{a}$ Exp. & Airmass & ${ }^{b}$ Lat. & ${ }^{b}$ Lon. & ${ }^{c}$ Dia. & ${ }^{d}$ Phase & Cal. Star \\
\hline 2006-01-15 13:42 & Kbb & 400 & 1.17 & -18.2 & 114.9 & 0.87 & 1.52 & HIP 53721 \\
2006-04-17 06:16 & Kbb & 360 & 1.04 & -19.8 & 36.3 & 0.79 & 6.25 & HIP 59271 \\
2007-01-29 12:29 & Kbb & 300 & 1.03 & -13.2 & 18.3 & 0.86 & 1.49 & HIP 59271 \\
2008-02-22 07:18 & Kbb & 360 & 1.58 & -8.1 & 148.9 & 0.86 & 0.34 & HIP 51213 \\
2008-03-25 05:32 & Kbb & 360 & 1.38 & -9.2 & 153.5 & 0.84 & 3.25 & HIP 51213 \\
2008-12-07 14:24 & Kbb & 360 & 1.26 & -0.8 & 179.4 & 0.76 & 6.01 & HIP 51213 \\
2010-01-04 14:34 & Kbb & 360 & 1.11 & 5.0 & 40.1 & 0.77 & 5.89 & HIP 62376 \\
2010-01-29 13:51 & Kbb & 360 & 1.06 & 4.8 & 244.8 & 0.80 & 4.90 & HIP 62376 \\
2011-01-10 15:05 & Kbb & 360 & 1.14 & 10.3 & 124.8 & 0.75 & 5.89 & HIP 69658 \\
2011-03-15 13:36 & Kbb & 360 & 1.22 & 9.4 & 131.2 & 0.82 & 2.19 & HIP 69658 \\
2012-01-02 14:43 & Kbb & 360 & 1.51 & 14.8 & 254.2 & 0.71 & 5.55 & HIP 69658 \\
2013-04-06 14:26 & Kn3 & 300 & 1.53 & 18.8 & 202.1 & 0.80 & 2.28 & HIP 69974 \\
2013-04-06 13:48 & Kn2 & 300 & 1.35 & 18.8 & 201.5 & 0.80 & 2.28 & HIP 69974 \\
2015-07-25 07:14 & Kn3 & 360 & 1.35 & 24.1 & 60.8 & 0.75 & 5.23 & HIP 75939 \\
2015-07-25 05:53 & Kn2 & 360 & 1.26 & 24.1 & 59.5 & 0.75 & 5.23 & HIP 75939 \\
2015-07-28 06:04 & Kn3 & 360 & 1.27 & 24.1 & 127.5 & 0.74 & 5.35 & HIP 75939 \\
2015-07-28 05:56 & Kn2 & 360 & 1.26 & 24.1 & 127.3 & 0.74 & 5.35 & HIP 75939 \\
\hline
\end{tabular}

${ }^{a}$ Exposure time in seconds for individual FOV. Four exposures with the same integration time were used to obtain data over the entire disk, with the exception of 2008-12-07 when we used five exposures.

${ }^{b}$ Sub-observer latitude and West longitude at the start of the first exposure from JPL Horizons ephemerides.

${ }^{c}$ Apparent diameter of the solid surface. The observable atmosphere increases this size by $\sim 10 \%$.

${ }^{d}$ The Sun-Target-Observer (S-T-O) phase angle. 


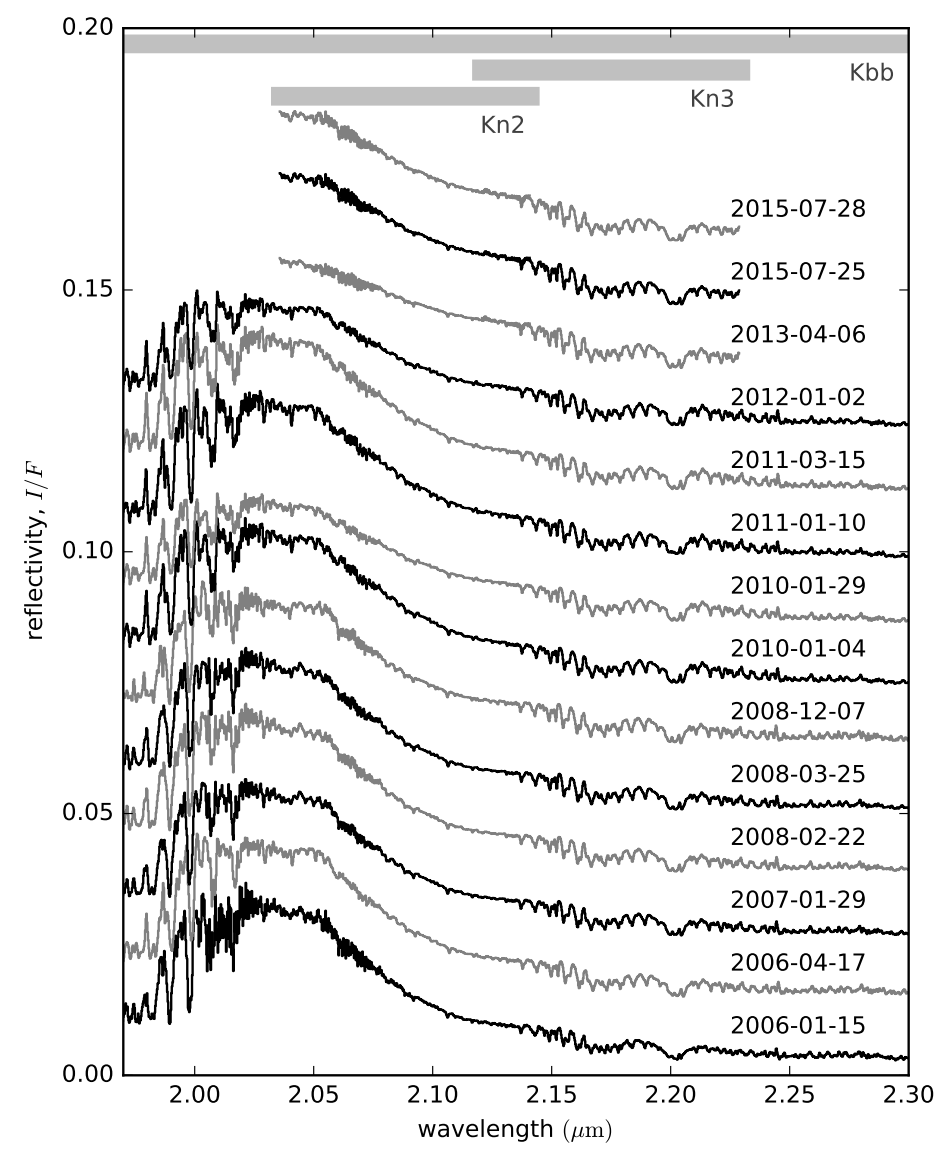

Figure 1: Disk-integrated spectra for all targets. Offset for clarity. The observations from 2013 and 2015 used narrow-band Kn2 and Kn3 filters, which cover a smaller spectra bandpass but benefit from a larger FOV that does not require mosaicking. 
$\mu_{e}$ for these low Sun-Titan-Observer phase angles, we discuss the path length through the atmosphere on Titan (or airmass) as generally characterized by an approximate $\mu \approx \mu_{0}$. A $2.018 \mu \mathrm{m}$ map of Titan (Barnes et al., 2007), updated with recent Cassini/VIMS measurements (Barnes, J. W., private communication) is to determine the surface albedo for each pixel. The limits in latitude and longitude for each pixel are calculated and the mean value of the rectangular footprint on the surface albedo map is used as input to the radiative transfer model, see Figure 2. We estimate a $10 \%$ systematic uncertainty in our surface reflectivity map, which is propagated through to our retrieved model parameters. With some constraint on the surface albedo, we can simulate a spectrum for a given viewing geometry that has only the properties of the haze as free parameters. The catalog of observations for the entire campaign is illustrated with parameters related to the viewing geometry and surface albedo in Figure 3.

\subsection{Aerosol scattering sensitivity tests}

Starting with a nominal model of the atmosphere, as measured by instruments on the Huygens probe, we perform a series of sensitivity tests to illustrate the spectral changes resulting from a variation in the haze optical depth. We make the assumption here that the DISR model at $2.0 \mu \mathrm{m}$ is a reasonable enough extrapolation of observations with maximum wavelength of $1.6 \mu \mathrm{m}$ to a be a valid starting point for our models. The sensitivity tests are used to inform the spectral fitting algorithm described below in Section 3.2, and to illustrate the limitations and uncertainties in the analysis. The tests are performed by adding a perturbation of $\delta \tau_{H}$ to the nominal haze opacity in a given layer. The difference between the simulated spectrum with the perturbation and the nominal spectrum is the spectral response, $\Delta I / F(\lambda)$, at each wavelength $\lambda$. The spectral response depends on the altitude of the haze perturbation, Figure 4. For example, increased haze opacity in the $53.5 \mathrm{~km}$ altitude layer of the model increases the reflectivity at all wavelengths (purple), whereas adding haze near the surface (red) does not change the spectrum at wavelengths longer than $2.15 \mu \mathrm{m}$. The wavelength region near $2.12 \mu \mathrm{m}$, which is generally thought to be diagnostic of clouds in the $20-40 \mathrm{~km}$ altitude range (e.g., Brown et al., 2002; Griffith et al., 2009; Ádámkovics et al., 2010), is also sensitive to haze opacity at other altitudes. This degeneracy means that a unique solution to the vertical structure of haze is not retrieved from an individual spectrum. The degeneracy is also part of the motivation for analyses with simplified scalings of the haze opac- 

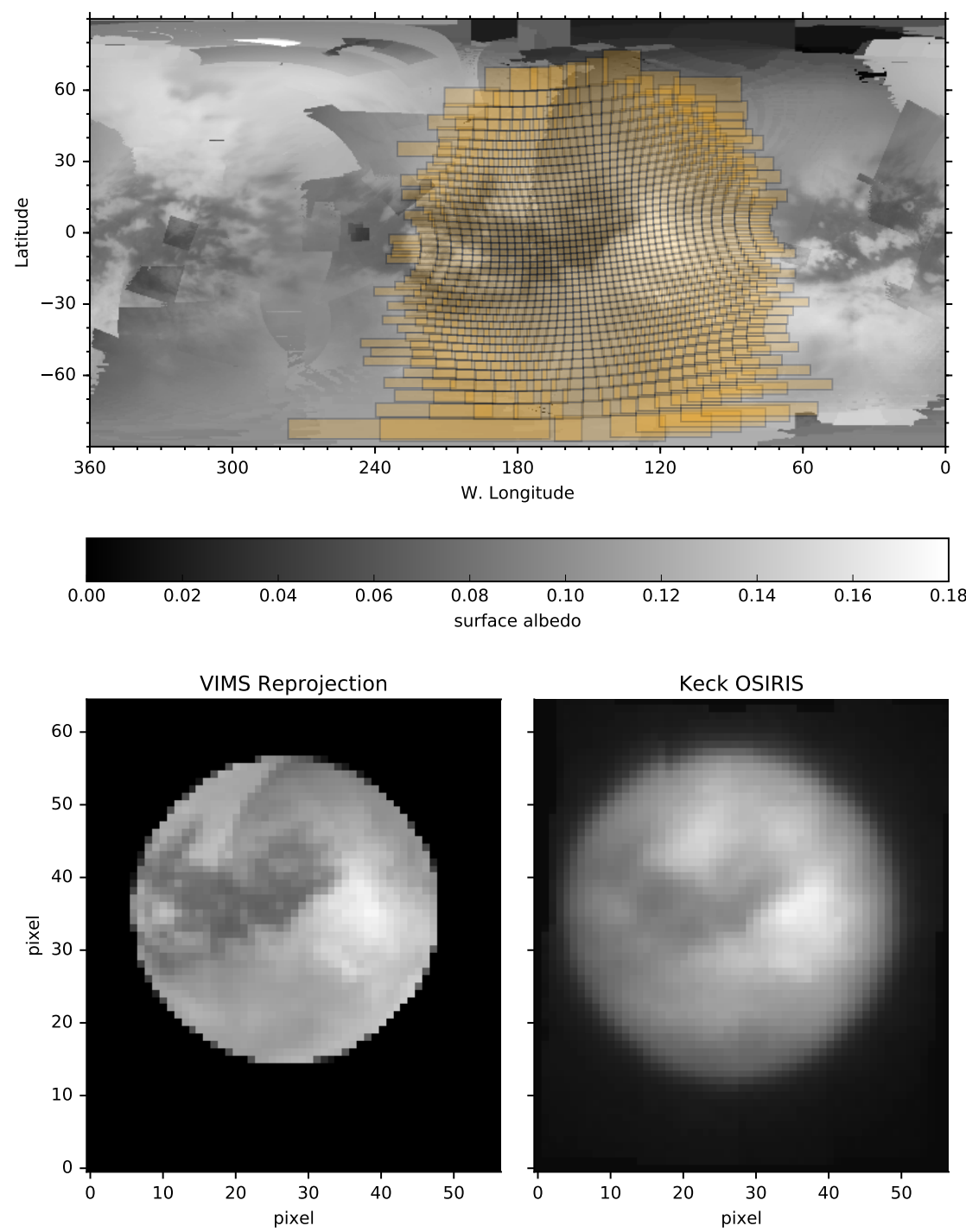

Figure 2: Surface reflectivity reprojection. An example of the surface albedo determination for each pixel in an observation is illustrated for the observations on 2008-02-22. A $2.018 \mu \mathrm{m}$ VIMS surface albedo map (top; updated from Barnes et al., 2007) is used to determine the mean surface albedo in the approximate footprint (orange squares) covered by a pixel. These values are used to generate a synthetic surface albedo for the observed disk (bottom left), which can be compared to observations at wavelengths that are sensitive to the surface (bottom right; 2.017-2.044 $\mu \mathrm{m}$ ). 

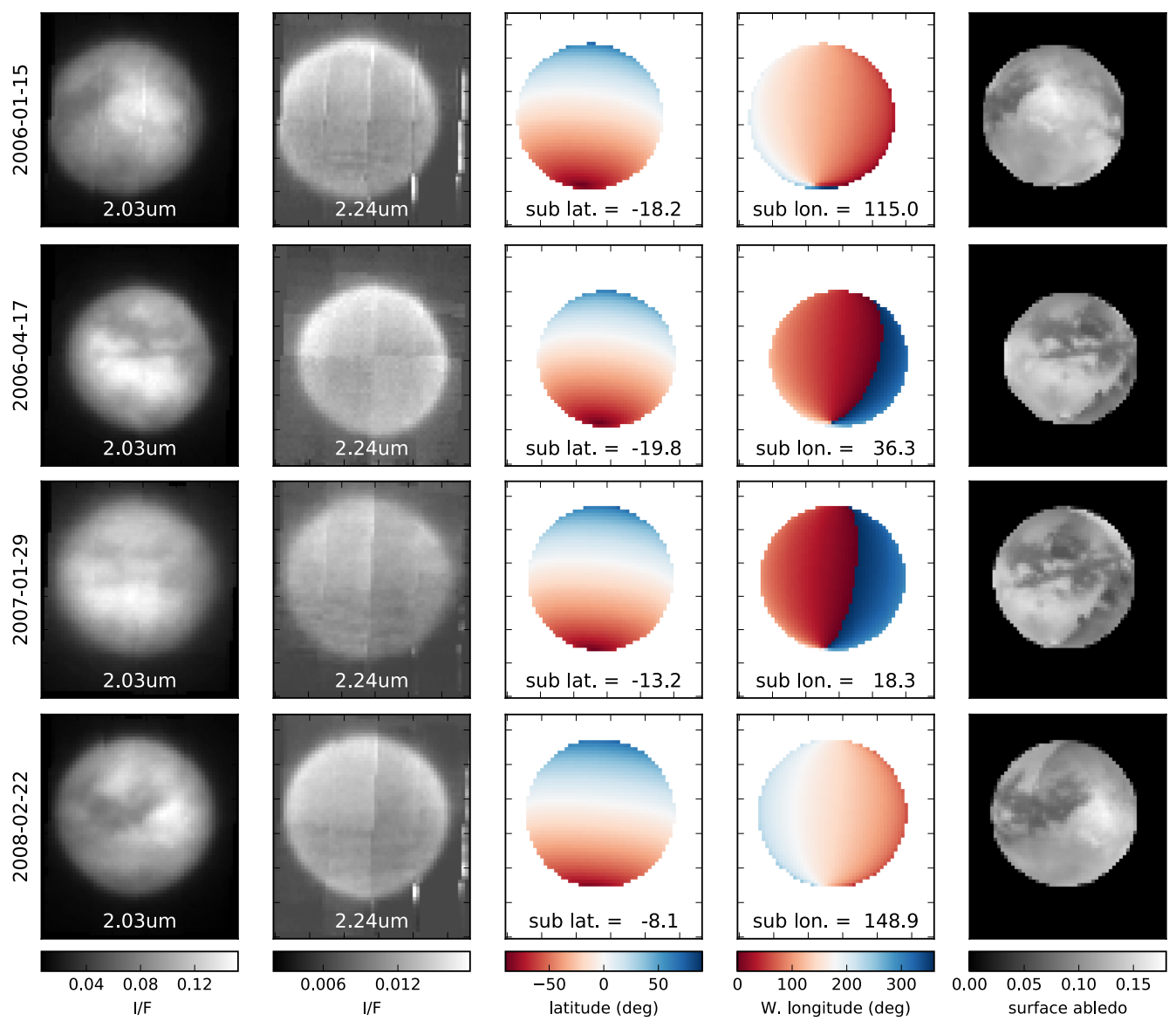

Figure 3: Catalog of observations. For each night of observations, two characteristic images that are sensitive to the surface $(2.017-2.044 \mu \mathrm{m})$ and stratosphere $(2.215-2.265 \mu \mathrm{m})$ are shown in grayscale. The color panels illustrate the latitude and longitude, and the rightmost panel in each set (grayscale) is the reprojected surface albedo map that corresponds to the viewing geometry. Mosaicking artifacts can be seen to be particularly prominent in 2010 and 2011, before being nearly completely eliminated in the observations between 2013 and 2015. Seams from the surface albedo map also show up in some of the reprojections. Uncertainty due to the surface albedo map is discussed in the text. 

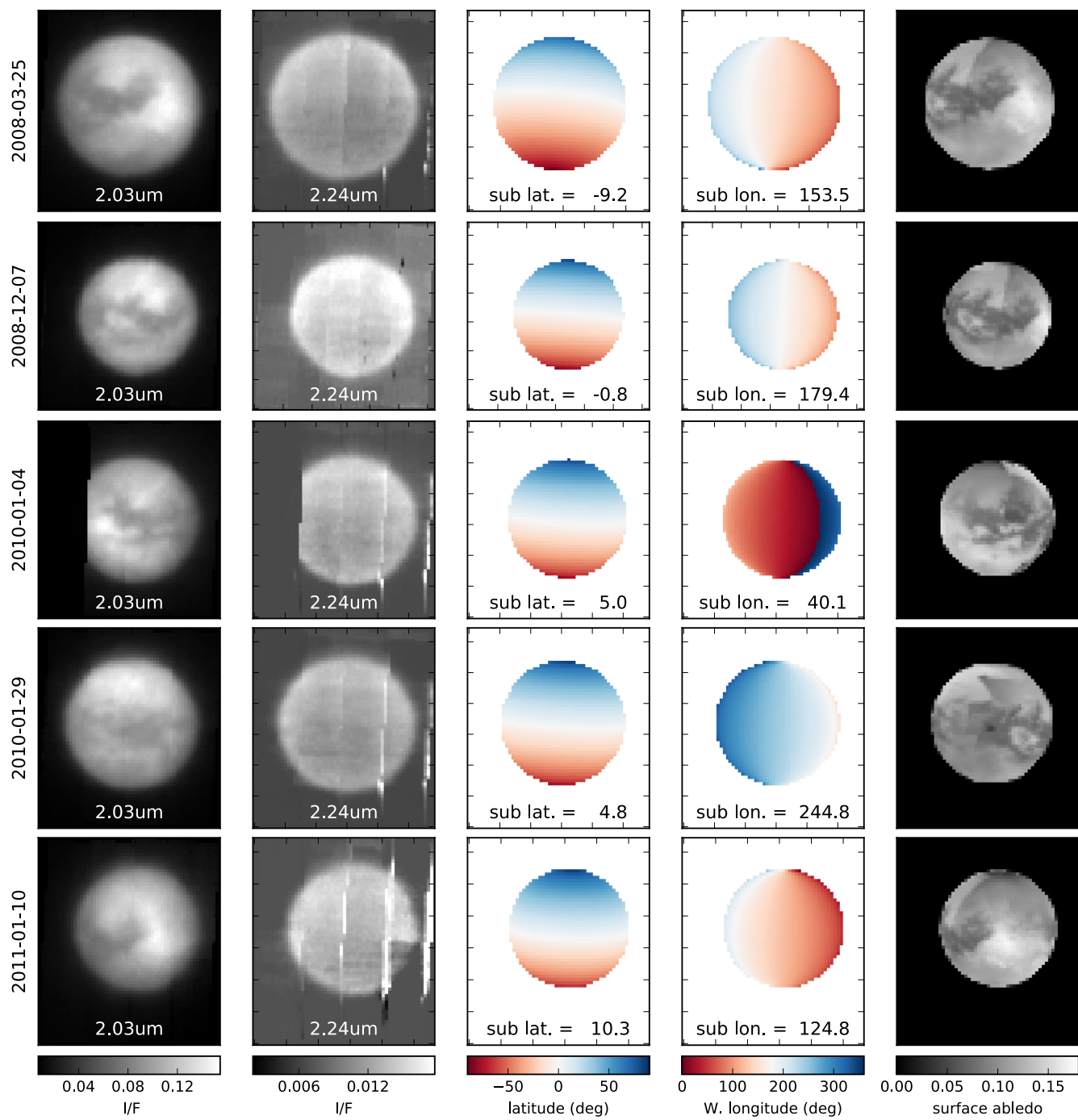

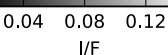

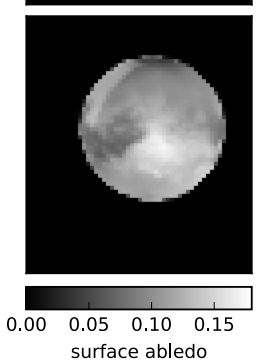

Figure 3: (continued) 

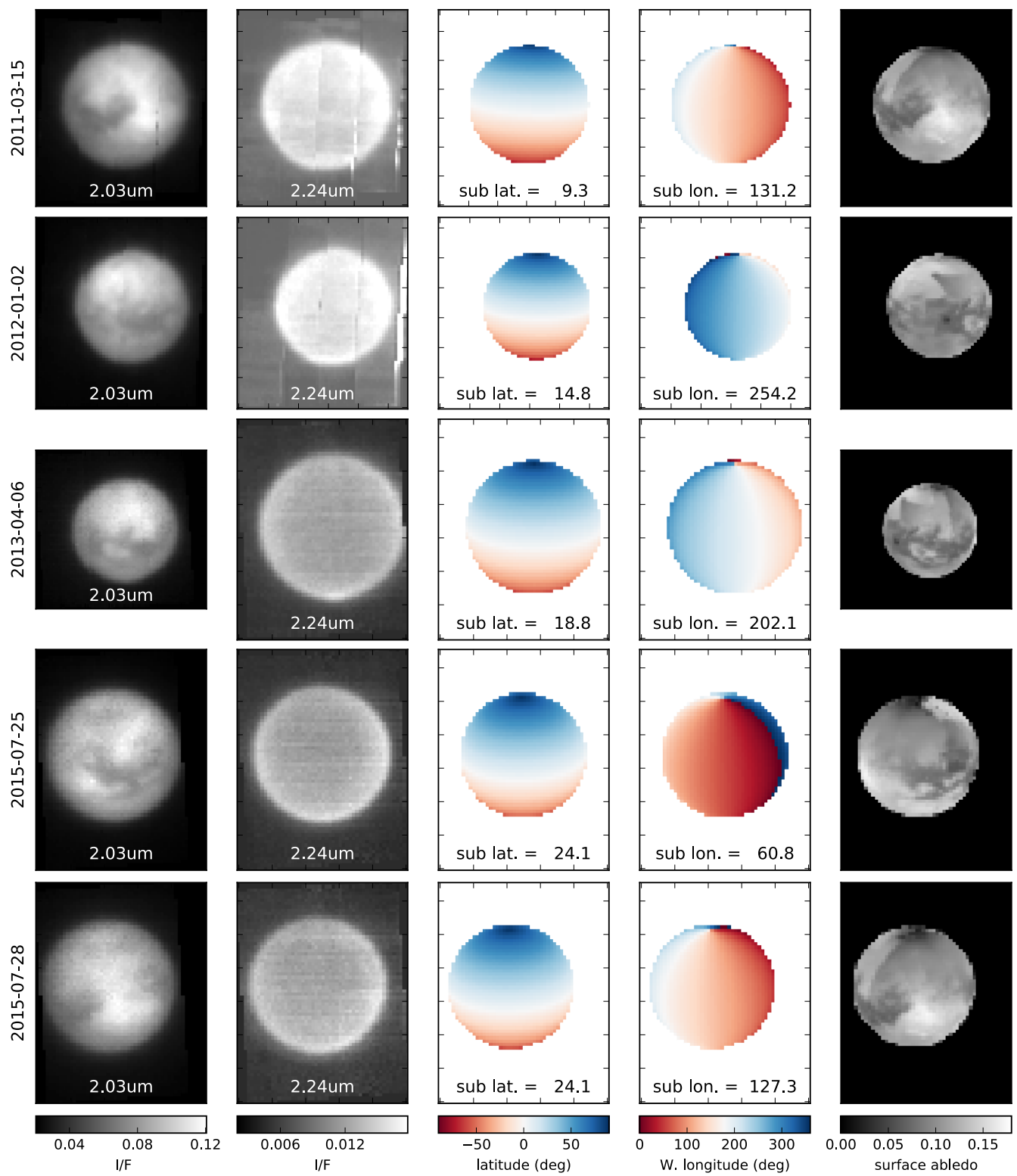

Figure 3: (continued) 
ity above and below a particular altitude in the atmosphere, e.g., Karkoschka (2016).

The altitude sensitivity tests can be used to select an altitude boundary in the model for the presribed changes to the haze vertical structure. We define two independent regions of "upper" and "lower" hazes separated at the altitude $z_{\text {crit }}$. The center of the K-band window near $2.16 \mu \mathrm{m}$ is the wavelength cutoff for sensitivity to hazes above and below $18.8 \mathrm{~km}$ (green dashed spectra in Figure 4). Adding haze opacity to the model below $\sim 20 \mathrm{~km}$ altitude does not impact the spectral fitting for $\lambda>2.16 \mu \mathrm{m}$, since $\Delta I / F(\lambda)=$ 0 . This means that roughly one half of spectral channels in the observations are sensitive to only the haze above $\sim 20 \mathrm{~km}$, while the other half are sensitive to both the hazes above and below. The model atmosphere is also setup such that roughly half the model layers are above and below this altitude (see Table 2 in Ádámkovics et al., 2016). The altitude boundary at $20 \mathrm{~km}$ altitude facilitates fitting spectra in the $2 \mu \mathrm{m}$ window but otherwise has no physical motivation.

Although the haze opacity is optically thin in each layer of the model, we test for non-linearity in the spectral response to $\delta \tau_{H}$ by comparing $\Delta I / F$ for values of $\delta \tau_{H}=0.1$ and 0.3 . The similarity of the panels on right and left sides of Figure 5 demonstrates that for an optically thin increase in haze opacity the magnitude of spectral response is proportional to $\delta \tau_{H}$. Indeed, there are no significant variations in the shape of the spectral response for the two cases shown in Figure 5. However, this is only demonstrated for an optically thin example, and would not necessarily remain true for optically thick increases in haze opacity.

Although the shape of the spectral response does not depend on $\delta \tau_{H}$, it does depend on the slant-path through the atmosphere, as determined by the viewing geometry. Adding the same opacity at larger airmass (smaller $\mu$ ) means that the same opacity should result in a larger $\Delta I / F$ at some wavelengths. We confirm and quantify this picture by comparing the spectral response to $\delta \tau_{H}=0.1$ for two viewing geometries (Figure 6). Comparing the left and right columns in Figure 6 confirms that the spectral response is larger for a given opacity increase in the higher-altitude regions of the atmosphere. On the other hand, the difference in the spectral responses at wavelengths sensitivei to the lower atmosphere is smaller. 

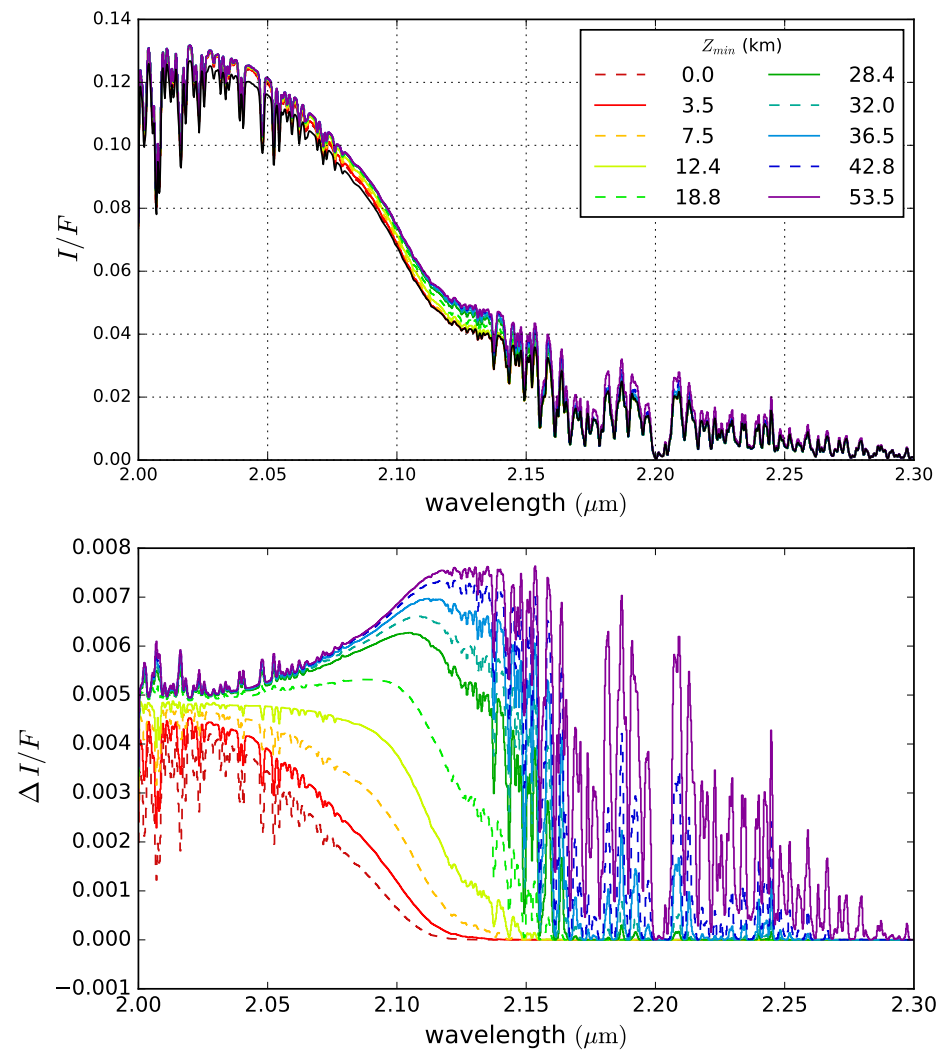

Figure 4: Altitude sensitivity of haze scattering. The nominal synthetic reflectivity spectrum (top; black) is compared to spectra where an increase in the haze opacity of $\delta \tau_{H}=0.1$ is added to 10 different altitude layers in the model (color spectra). The altitude at the bottom of the model layer $z_{\min }$ is specified in the legend. The spectral response $\Delta I / F(\lambda)$ (bottom) is the difference between the model reflectivity for two simulations; one with the added haze opacity $\delta \tau_{H}$ in one layer of model, as compared to the nominal reference model, for a given wavelength, $\lambda$. 

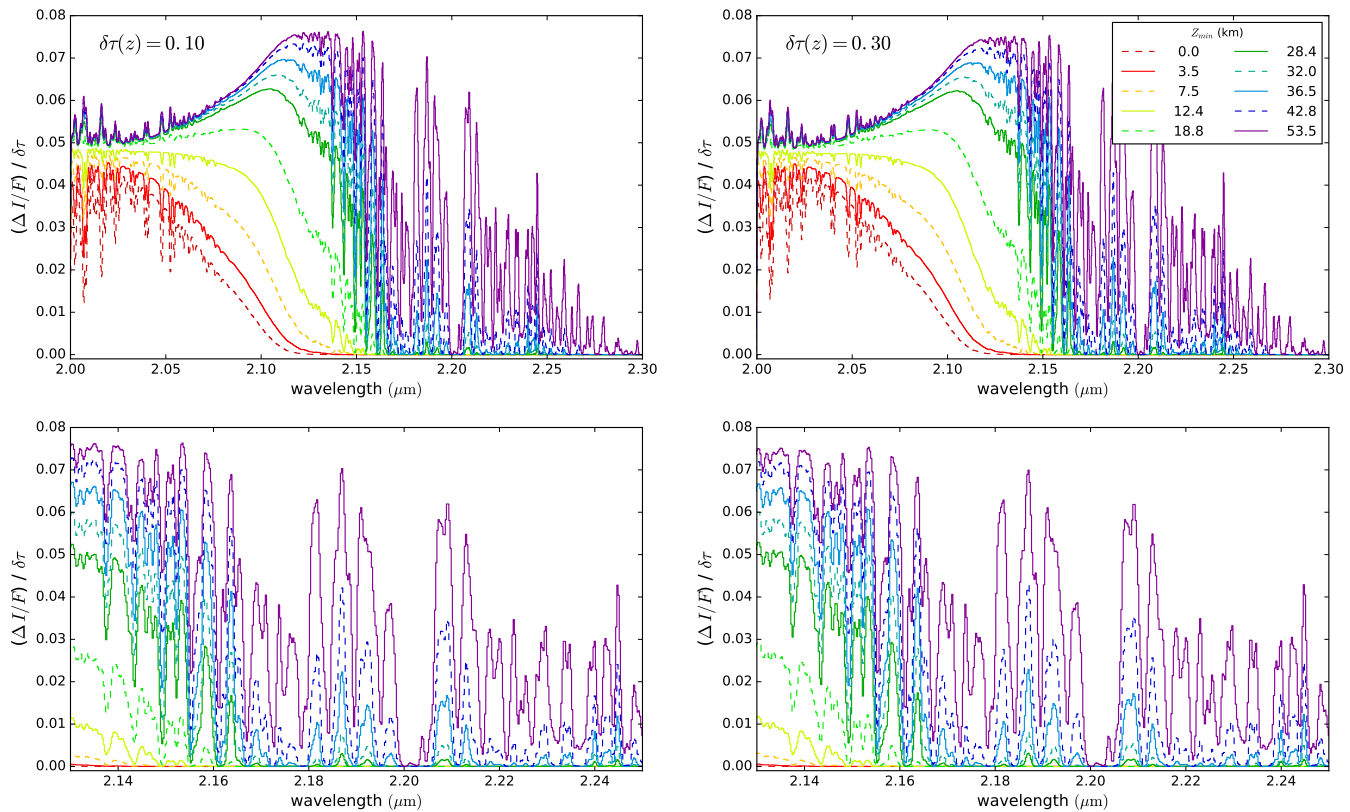

Figure 5: Optical depth sensitivity of haze scattering. The spectral response plotted in the bottom panel of Figure 4 is shown here, but in this case normalized by the magnitude of the optical depth increase, $\delta \tau_{H}=0.1$ (top left). The spectral response for a $3 \times$ larger increase of $\delta \tau_{H}$ is also show (top right). The similarity of the expanded spectral regions in the bottom row illustrates the linear spectral response to an optically thin increase in haze scattering at various altitudes. 

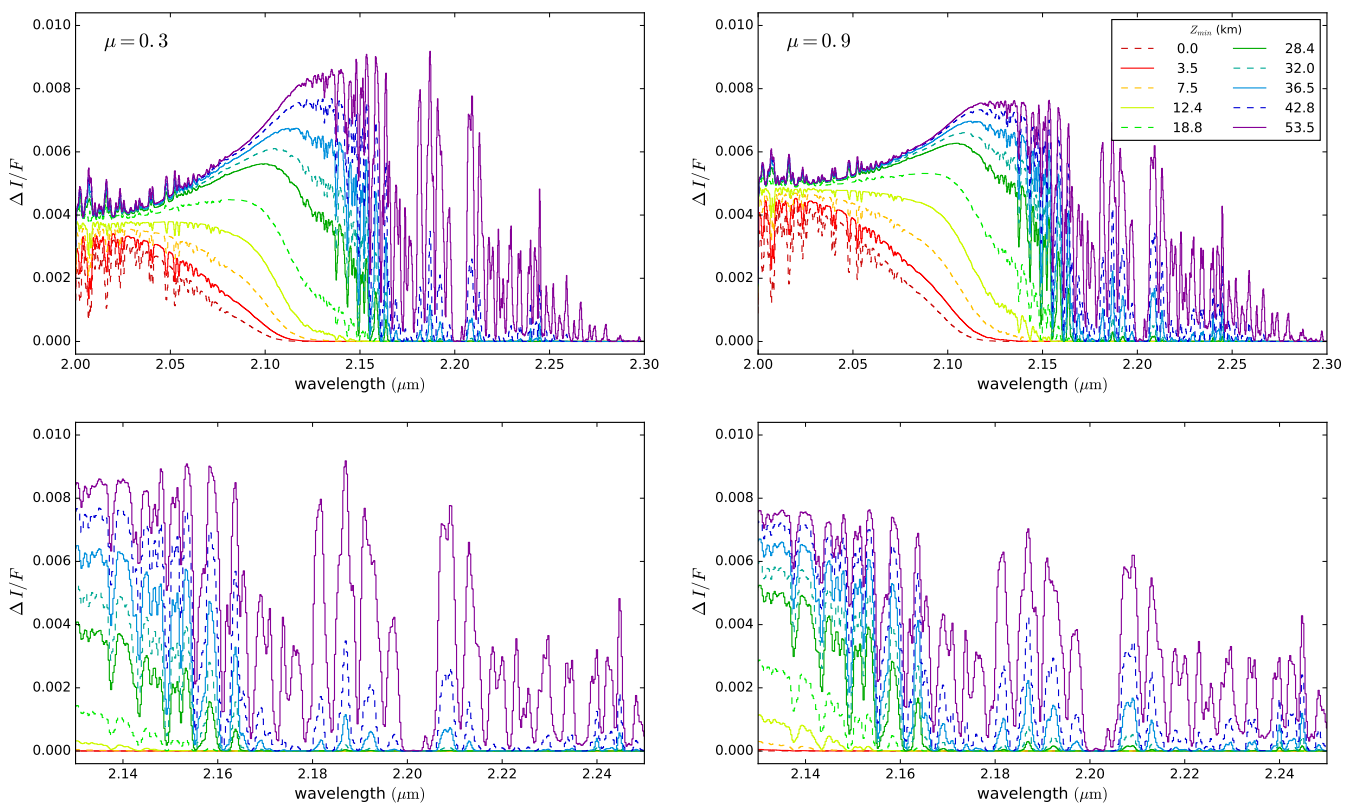

Figure 6: Airmass sensitivity of haze scattering. The spectral response $\Delta I / F(\lambda)$ due to an increase in the haze opacity by $\delta \tau_{H}=0.1$ at various altitudes is shown by the colored spectra for an airmass of $\mu=0.3$ (left) and compared with the corresponding spectra for and airmass of $\mu=0.9$ (right). In the case of the larger airmass (smaller $\mu$ ), $\Delta I / F$ can be up $\sim 20 \%$ larger or smaller, depending on the altitude of the haze increases. For example, increasing the haze in the $53.5 \mathrm{~km}$ layer of the nominal model at low airmass $(\mu=0.9$ right) corresponds to $\Delta I / F(2.19)=0.007$, whereas at larger airmass $(\mu=0.3$; left $)$, there is a larger $\Delta I / F(2.19) \sim 0.009$. The opposite trend occurs for a haze increase in the $28.4 \mathrm{~km}$ layer of the model near $2.13 \mu \mathrm{m}$, where the response is smaller at large airmass. The illustrative case of $\mu=0.3$ is an extreme example used to bracket our modeling uncertainty, and we do not consider $\mu<0.68$ when fitting observations. 


\subsection{Spectrum fitting}

Fitting each individual spectrum in the observations with the PyDISORT radiative transfer model is computationally expensive. If each of the 26.2 million (spatial and spectral) datapoints from the observations could be fit in $1 \mathrm{~s}$, it would take 10 months to fit all of the observations. However, the computing time for running a single monochromatic channel with PyDISORT on a standard 24-core workstation is a few seconds, and it takes (at least) a few iterations of the calculation for a fitting algorithm to converge. This makes fitting of the entire dataset of Keck OSIRIS observations unfeasible with a standard workstation.

In order to minimize the amount of data that is simulated, and reduce the number of radiative transfer calculations that are required, we (1) average the spectra in latitude bands on Titan, thereby integrating across longitudes that are assumed to have similar haze properties, and (2) reduce the total number of channels in the spectra that are fit during the optimization, by assuming that multiple channels redundantly sample the same altitude regions of the atmosphere. The fit is confirmed with a full spectrum calculation after the parameter optimization is complete. These two approximations are described in greater detail below.

Latitude band integration: The hazes on Titan can vary significantly with latitude, but much less so with longitude (Smith et al., 1981, 1982; Lorenz et al., 2001; Rannou et al., 2000; de Pater et al., 2006; Anderson et al., 2008; Lorenz et al., 2010; Penteado et al., 2010; de Kok et al., 2010; Rannou et al., 2010; Larson et al., 2015). We take advantage of the symmetry over longitude to combine spectra and reduce the number of calculations needed, while also improving the signal to noise ratio of the observation being simulated. However, there is a significant variation in the spectral response with airmass (Figure 6), and spectra from various paths through the atmosphere are obtained from within a latitude region. Therefore, we evaluate the number of spectra that can be combined without introducing significantly increased uncertainty in the combined spectra due to variation in the viewing geometry. For example, in the observations on 2008-02-22 there are 40 spectra in the latitude region bounded by $26.9^{\circ} \mathrm{S}$ and $29.8^{\circ} \mathrm{S}$. In the Supplementary Material we illustrate that the systematic uncertainty introduced by combining the 20 spectra at lowest airmass, with $\mu>0.8$, is negligible.

The integration over latitude band regions is performed over the entire disk. Starting from the south pole, the latitude limit that encompasses 40 pixels is determined (e.g., from $90^{\circ} \mathrm{S}$ to $66.1^{\circ} \mathrm{S}$ on $2008-02-22$ ), and the band 
integration is performed by considering the 20 spectra with the smallest airmass. The excluded pixels occur near the East and West limb, with the low airmass pixels coming from near the center of the disk. The process is repeated northward. The latitude band regions near the north and south limb have a low median $\mu$, which corresponds to a high airmass that is challenging to treat in the pseudo-spherical radiative transfer model. In the subsequent analysis of retrieved haze parameters, the latitude band regions that do not have a median $\mu>0.7$ are ignored.

The largest airmass that is considered in this work is 1.48, corresponding to a viewing geometry with $\mu=0.68$ and solar zenith angle of $48^{\circ}$. In general, most datasets have a maximum airmass of $\sim 1.3$, such that the pseudo-spherical correction is an applicable correction to the radiative transfer calculation.

Spectral region selection: Wavelengths in the $2 \mu \mathrm{m}$ window are sensitive to various altitudes throughout the atmosphere and surface, e.g., see contribution functions in Figure 6 in Ádámkovics et al. (2006). For the $2 \mu \mathrm{m}$ window in general, the fainter regions of low $I / F$ (with large gas opacity) are sensitive to higher in the atmosphere, whereas bright regions (with lower gas opacity) are sensitive to the surface. It can be approximated that regions of similar $I / F$ are roughly sensitive to similar altitude regions. Due to this redundancy, we do not fit all 1665 spectral channels in the K-band. Instead, we pick seven wavelength windows listed in Table 2 that sample altitudes throughout the atmosphere, using a total of 104 OSIRIS spectral channels. By considering this subsample of 104 channels from the integrated spectra for $\sim 29$ latitude bands, we reduce the total number datapoints being fit by a factor of $\sim 800$ for each observation.

Parameter optimization: We fit each spectrum with a model that includes two free parameters; one parameter $f_{S}$ scales the haze opacity above a critical altitude $z_{\text {crit }}=20 \mathrm{~km}$ and a second parameter $f_{T}$ scales the haze opacity below this altitude. Since the surface albedo is determined from the surface reflectivity map, and we assume the temperature and methane abundance profiles measured by instruments on Huygens, there are no other unconstrained parameters. Starting from the standard reference spectrum, a Levenberg-Marquardt (LM) optimization is used to simultaneously fit $f_{S}$ and $f_{T}$. For simplicity in our approximate approach, we consider $z_{\text {crit }}$ to be fixed. A higher (lower) value for $z_{\text {crit }}$ means that the total column of haze opacity being scaled by each parameter is different and would result in a systematically smaller (larger) value retrieved value for $f_{T}$. 
Table 2: Spectral Regions for RT Model Optimization

\begin{tabular}{cc}
\hline Wavelength $(\mu \mathrm{m})$ & Channels \\
\hline $2.042-2.045$ & 12 \\
$2.072-2.074$ & 8 \\
$2.092-2.094$ & 8 \\
$2.101-2.105$ & 16 \\
$2.132-2.135$ & 12 \\
$2.141-2.145$ & 16 \\
$2.182-2.186$ & 16 \\
$2.200-2.204$ & 16 \\
\hline
\end{tabular}

Although the shorter wavelengths in the K-band window are sensitive to changes in both parameters, the altitude sensitivity test (above) suggests that a two-parameter optimization of the model with a boundary at $20 \mathrm{~km}$ is not degenerate, since the longer wavelengths can be used to independently constrain $f_{S}$. In practice we us scipy.optimize.curve_fit with a function that is a wrapper to a PyDISORT calculation for the 104 channels in the seven wavelength windows. The LM optimization usually converges with $\sim 10$ spectral calculations. Once $f_{S}$ and $f_{T}$ have been optimized for the spectrum from a latitude band region, a full spectrum over the 1665 channels is calculated for direct comparison and validation.

\section{Results}

The best fit spectra for a range of latitude band regions are compared with the observations from 2008-02-22 in Figure 7. The optimized values for $f_{S}$ and $f_{T}$, along with the mean surface albedo in the latitude band, $\bar{A}_{S}$, are shown for each panel. The models generally reproduce the observations for the spectra at $\lambda>2.14 \mu \mathrm{m}$. This is primarily because these wavelengths are insensitive to the surface albedo and are sufficiently modeled with a singleparameter scaling of the haze above $20 \mathrm{~km}$. It is important to note that as $f_{S}$ varies the haze in the uppermost layers of the model, which $\lambda>2.14 \mu \mathrm{m}$ are most sensitive to, this spectral region and the retrieved $f_{S}$ is not particularly sensitive to $z_{\text {crit }}$. However, as described above, the retrieved value of $f_{T}$ does depend on the $z_{\text {crit }}$. The largest model error at $\lambda>2.14 \mu \mathrm{m}$ occurs 
in the two latitude band regions nearest the north and south limb, where the simulated spectra overestimate the $I / F$ near $2.20 \mu \mathrm{m}$. The overestimate in the spectral region with the smallest $I / F$, sensitive to the highest altitudes in the atmosphere, demonstrates the limitations of the pseudo-spherical correction in the plane parallel model. However, since the latitude regions near the limb have a broad range of airmass, we've already excluded them from the analysis (Section 3.2) and do not consider the retrieved values of $f_{T}$ and $f_{S}$ in the analysis below.

At shorter wavelengths $(\lambda<2.14 \mu \mathrm{m})$, the most significant discrepancy between the simulated spectra and observations occurs near $2.09 \mu \mathrm{m}$ (Figure 7). This wavelength region is sensitive to haze variation throughout the atmosphere (e.g., Figures 4 and 6), and therefore decreasing $f_{T}$ in order to decrease $I / F$ near $2.09 \mu \mathrm{m}$ would also decrease $I / F$ near $2.05 \mu \mathrm{m}$ and degrade the fit. The limitation in the spectral fitting arises from only using two parameters to model the variation in the haze distribution. Using additional free parameters would improve the fit, but would lead to degeneracies among the haze scaling parameters. Additional free parameters would also significantly increase the calculation time of the LM parameter optimization.

One possible source of error is a systematically underestimated surface albedo from the brightest regions of the VIMS albedo map. Increasing the surface albedo that is used in the simulations would result in a fit with a decreased $f_{T}$. Increasing the surface albedo and decreasing $f_{T}$ would improve the fit to the observations at both $2.05 \mu \mathrm{m}$ and $2.09 \mu \mathrm{m}$. Alternatively, the gas opacity in this wavelength region may be underestimated in the model, or the haze single scattering albedo in this wavelength region may be too high. Both the gas opacity and the haze scattering albedo are assumed to be uniform at all locations.

The retrievals of $f_{S}$ and $f_{T}$ that have been illustrated for 2008-02-22 are performed for each of the observations. The meridional trend in haze, as represented by the best fit parameter $f_{S}$, is characterized by a seasonally variable linear gradient in haze toward the North pole, together with a seasonally variable decrease in opacity poleward of $20^{\circ}$ (Figure 8 ). The magnitude of the linear gradient toward the North increases with time over the timespan of the observations from 2006 through 2013, before starting to decrease. The overall trend is complicated by the poleward decrease in opacity. While the decrease near the limb raises suspicions of a modeling or retrieval artifact, there are a few considerations that suggest the decreases may be real: (1) in nearly all of the observations, with the exception of 2010-01-29, the decrease 

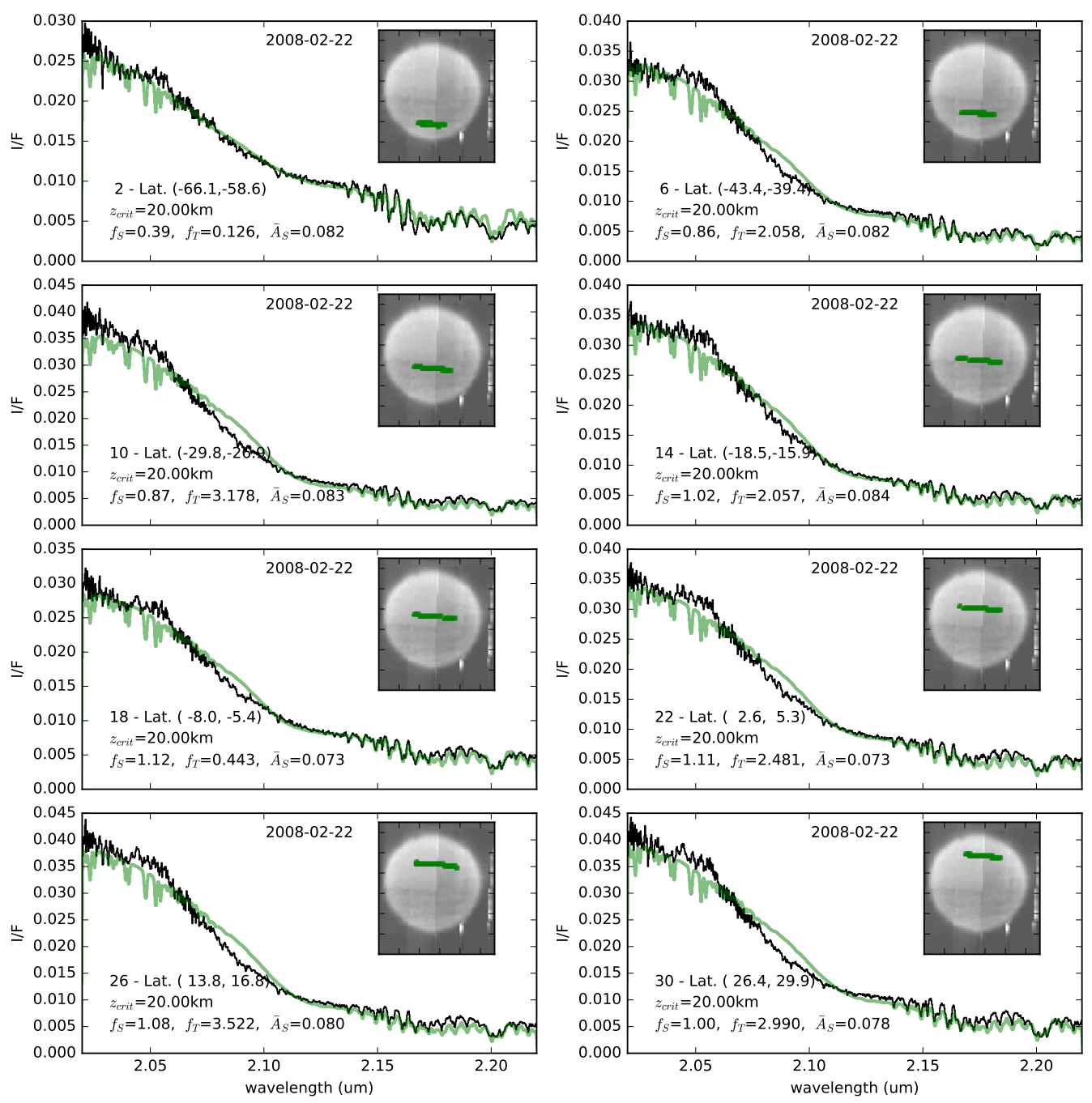

Figure 7: Two-parameter model optimization for $f_{S}$ and $f_{T}$. The best fit models (green spectra) and the observations (black spectra) that are integrated over a particular latitude band (green pixels in inset image) are illustrated for each latitude band (panels). The observations from 2008-02-22 are shown. For each panel, the latitude range for the observed spectrum is given in parenthesis. The best fit haze scale factors; $f_{S}$ above $20 \mathrm{~km}$ and $f_{T}$ below $20 \mathrm{~km}$, are listed in each panel with the median surface albedo in the region $\bar{A}_{S}$. The inset image of is integrated over $2.21-2.26 \mu \mathrm{m}$. 
is either absent or occurs asymmetrically toward one limb. A geometric effect whereby the retrieval systematically underestimates $f_{S}$ near the limb is anticipated to result in symmetric underestimates at both the North and South edges of the disk. (2) The decease in $f_{S}$ toward the limb varies among the observations, sometimes occurring in the South (2011) and sometimes occurring in the North (2006). A geometric artifact, even if asymmetric for some reason, would not be seasonally variable. (3) the latitude regions that are closest to the limb, where retrieval artifacts due to model limitations at high airmass are most significant, have been excluded and are not plotted in Figure 8 . The poleward decrease in $f_{S}$ may indeed be an indication of the formation of features like the polar collar (Smith et al., 1981; Lorenz et al., 2001; de Pater et al., 2006).

The plot of the $f_{T}$ vs latitude (Figure 9) demonstrates a general correlation between the surface albedo and the lower atmospheric haze. Along with this correlation, there are noteworthy changes in the tropospheric haze. For example, In 2006 there was a 'bulge' in southern tropospheric haze, with the peak of the bulge shifting southward by $20^{\circ}$ latitude over three months. Features in these plots are likely due to uncertainties in the absolute value of the surface albedo map. Since it is difficult to remove a uniform contribution from atmospheric scattering to the entire map, there remains some uncertainty in the surface reflectivity. If the surface reflectivity is systematically (over-) underestimated, then the optimized value for $f_{T}$ will be (under-) overestimated. In the case of the 2013 observations, the limits of the absolute flux calibration are reached due to cloudy observing conditions. The best fit parameter for the $f_{T}$ is the complete removal of haze below $20 \mathrm{~km}$, however, the observations are consistent with uniform tropospheric haze.

There are large negative excursions from the otherwise relatively smooth meridional trends in the retrieved values for $f_{T}$ (Figure 9) on 2008-02-22, 2008-03-25, 2011-01-10, and 2011-03-15. On each of the dates, the small values in $f_{T}$ occur between the equator and $10^{\circ} \mathrm{S}$ latitude. The sub-longitude on these dates are $149^{\circ} \mathrm{W}, 153.5^{\circ} \mathrm{W}, 125^{\circ} \mathrm{W}$, and $131^{\circ} \mathrm{W}$. This suggests that there is a geographic area constrained in both latitude and longitude where the haze scattering opacity needs to be completely removed in order to fit the observations. On the other hand, it has been suggested that the methane opacity in this region, corresponding to the Xanadu region, might be preferentially enhanced (Ádámkovics et al., 2007, 2009). If this is the case, then the variation in methane opacity would allow for more uniform variation in the haze, as is seen at other longitudes. In other words, by invok- 
ing an increased methane opacity near Xanadu, the fitting algorithm would not converge on a decrease $f_{T}$, and the smoother latitudinal variation that is observed on other dates would occur in all cases. An alternate explanation is that the brightness of the surface albedo near Xanadu in the VIMS maps is overestimated. Overestimating the surface albedo would result in artificially bright simulated spectra, which would result in the optimization returning small (or zero) values for $f_{T}$. However, this somewhat contradicts the interpretation of the shape of the simulated spectra, in particular the (under)overestimate of $I / F$ at (2.04)2.09 $\mu \mathrm{m}$, which could be explained by a brighter surface albedo map. An interpretation that we cannot rule out is that perhaps both the overall reflectivity of the VIMS surface albedo map is too low while the reflectivity of Xanadu specifically is too high.

\section{Discussion}

The hazes on Titan have been studied with extensive observations (e.g., Smith et al., 1981, 1982; Coustenis and Bezard, 1995; Hutzell et al., 1996; Lorenz et al., 2001; Gibbard et al., 1999; Rannou et al., 2000; Tomasko et al., 2005; Ádámkovics et al., 2006; Anderson et al., 2008; Rannou et al., 2010; Lorenz et al., 2010), and models of circulation predict that the winds in the atmosphere seasonally redistribute the haze (e.g., Rannou et al., 2002; Larson et al., 2015). Recently presented analysis of HST-STIS observations by Karkoschka (2016) suggest that the observations of haze can be wellapproximated by a sinusoidal variation in haze components above and below $\sim 100 \mathrm{~km}$, with a phase lag between the two components and a meridional dependence in the magnitude of the sinusoid variation. Our results suggest that such a model may be an oversimplification of the haze variation. We observe meridional structure in the haze that is often characterized significant asymmetries and abrupt variations in the opacity of haze with latitude.

Penteado et al. (2010) studied VIMS datasets covering the timespan of July 2004 - March 2007 simulating spectra in the $0.4-1.6 \mu \mathrm{m}$ range. The shorter wavelengths (relative to this study at $2 \mu \mathrm{m}$ ) are sensitive to higher altitudes in the atmosphere. Penteado et al. (2010) fit their VIMS spectra with both haze opacity and scattering albedo variation above $80 \mathrm{~km}$. They find that the haze opacity above $80 \mathrm{~km}$ increases from $\sim 0.9$ near $60^{\circ} \mathrm{S}$ to 1.1 at $40^{\circ} \mathrm{N}$ and that the scattering albedo decreases by $\sim 20 \%$ toward the North over this region. We have temporal overlap with three of our datasets, (2006-01-15, 2006-04-17, and 2007-01-29), and can make a comparison to 

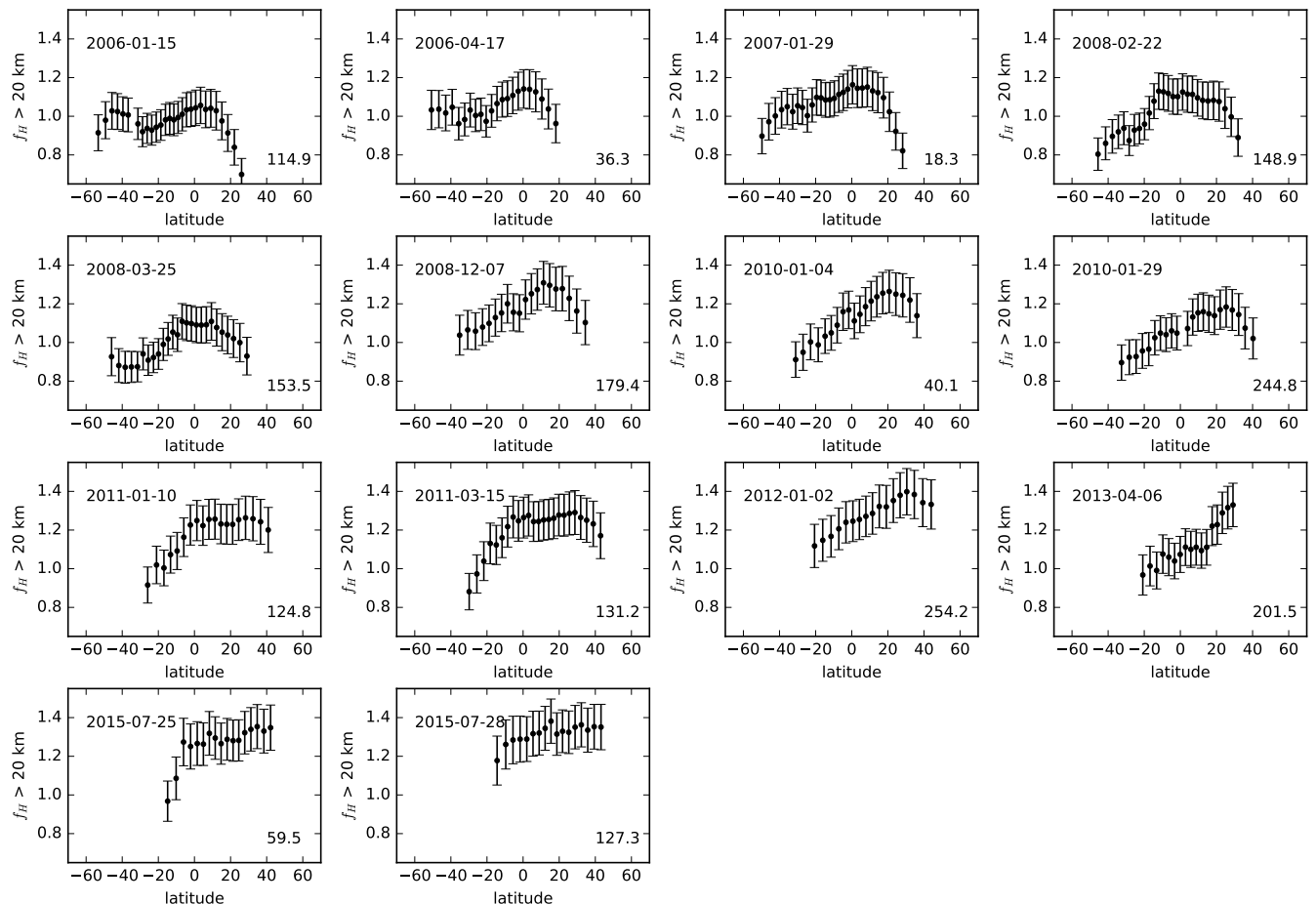

Figure 8: Meridional variation in the haze above $20 \mathrm{~km}$ altitude. LM optimization is used to determine the scale factor $f_{S}$, for each latitude region in each of the observations (panels). The date is indicated in the top left and the sub-observer West longitude indicated in the bottom right of each panel. 

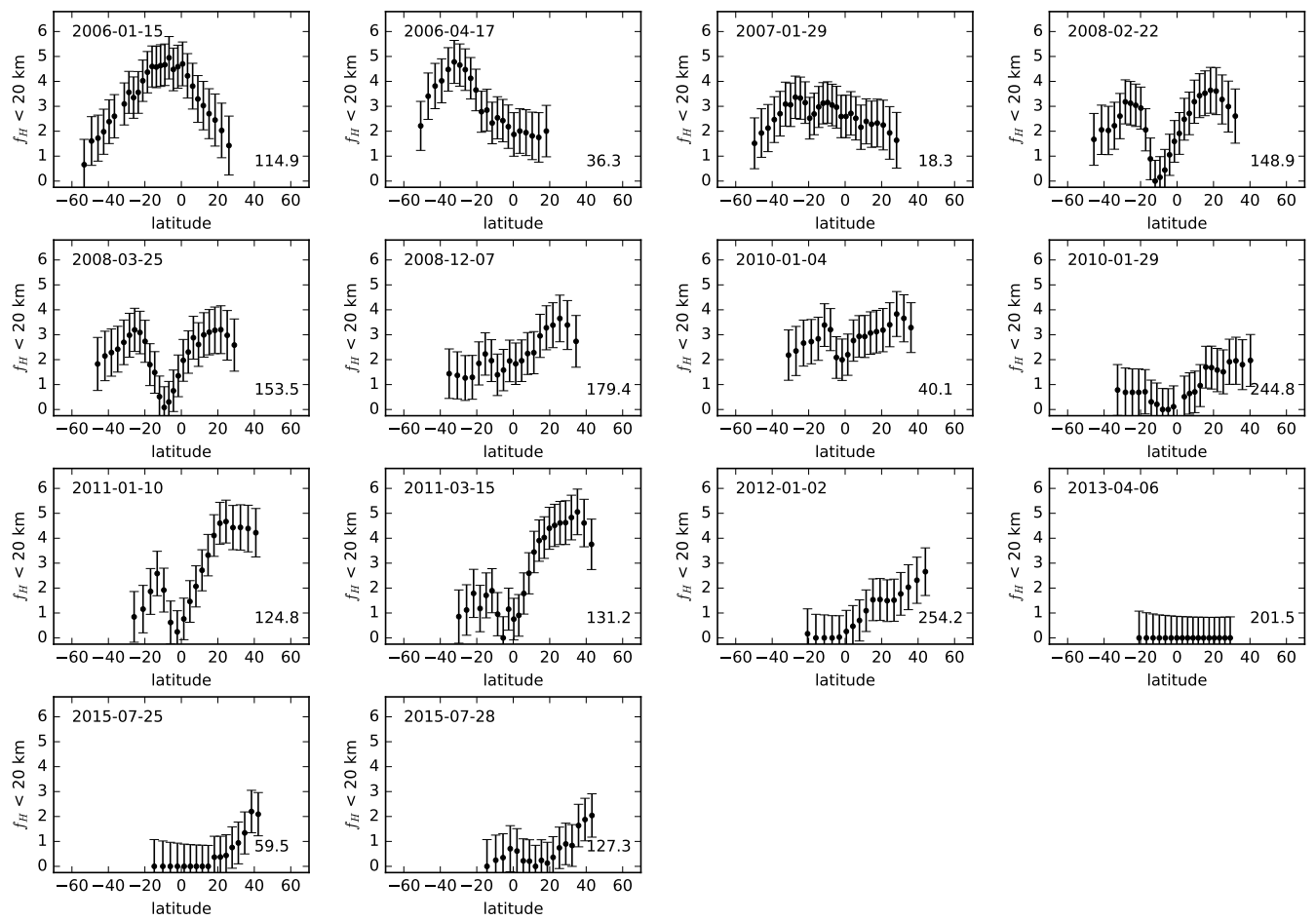

Figure 9: Meridional variation in the haze below $20 \mathrm{~km}$ altitude. LM optimization is used to determine the scale factor $f_{T}$, for each of the observations (panels). The date is indicated in the top left and the sub-observer West longitude indicated in the bottom right of each panel. 
their results, where both studies consider the case of uniform methane and evaluate the variation in haze.

Comparing Figure 16 of Penteado et al. (2010) (covering 2004-2007) to Figure 8 of this work (for individual dates), it can be noted that the multiplicative scale factors in both studies are generally in agreement: $\sim 0.9$ near $50^{\circ} \mathrm{S}$ and increase northward to a factor of $\sim 1.1$ near $20^{\circ} \mathrm{N}$. An interesting bump in the haze opacity near $60^{\circ} \mathrm{S}$ in the Penteado et al. (2010) is also reproduced in our observations in 2006, but is gone by 2007. And important difference is the drop in haze opacity that we observe north of $\sim 10^{\circ}$ N. Some amount of the discrepancy may be due our assumption of uniform singlescattering albedo. Penteado et al. (2010) implement a decrease in scattering albedo toward the north that could account for the some of the decrease in reflected flux that we simulate with decreased haze opacity. On the other hand, the decrease in haze opacity with latitude that we retrieve is steeper than the decrease in scattering albedo retrieved by Penteado et al. (2010).

Our observations and analysis are complementary to recent HST-STIS analysis (Karkoschka, 2016) in many ways, including the wavelength region and resolution, as well as the timing and time-span of the observations. Table 3 lists the characteristics of the datasets presented here and by Karkoschka (2016). In addition to the characteristics of the datasets, the method of analysis is also complementary. The large number of datapoints in both studies, and the calculation time required for generating simulated spectra, means that some method of reducing the total number of datapoints need to be invoked. While Karkoschka (2016) use a principle components analysis to identify the relevant features of the dataset, we use a two-parameter LM optimization to fit the spectra. Rather than avoiding the spectral bands that are most sensitive to the surface, we include them and model the entire K-band.

A quantitative comparison to the work of Karkoschka (2016) should not simply compare haze scale factors because of different choices how the hazes are varied in the radiative transfer models. One important consideration is the altitude region considered; above and below $20 \mathrm{~km}$ here, vs above $150 \mathrm{~km}$ and below $80 \mathrm{~km}$ in Karkoschka (2016). This is in addition to differences in the nominal haze model as well as wavelength sensitivities. Rather than compare derived haze opacity scale factors, we take a closer look at the corresponding $2 \mu \mathrm{m}$ optical depth variation. The nominal $2 \mu \mathrm{m}$ optical depths for the two models that are based on the DISR observations are shown in Figure 10. There is a haze extinction optical depth of, $\tau_{H}=0.13$ above $150 \mathrm{~km}$ 
Table 3: Comparison with HST analysis

\begin{tabular}{|c|c|c|}
\hline & This work & Karkoschka (2016) \\
\hline Instrument & Keck OSIRIS & $H S T-S T I S$ \\
\hline Spectrometer & $\mathrm{IFU}$ & slit \\
\hline Wavelength & $1.965-2.381 \mu \mathrm{m}$ & $0.530-1.020 \mu \mathrm{m}$ \\
\hline Plate scale & 20 mas/pixel & 50 mas/pixel \\
\hline$R=\lambda / \delta \lambda$ & 3,700 & 1,500 \\
\hline Obs. Start & $2006-01-15$ & 1997-11-03 \\
\hline Obs. End & 2015-07-28 & 2004-01-03 \\
\hline Datapoints & $26.2 \times 10^{6}$ & $\sim 10^{6}$ \\
\hline Number of Obs. & 14 & 5 \\
\hline \multicolumn{3}{|l|}{ Model } \\
\hline Spectral bands & 104 & 25 \\
\hline Latitude regions & 29 & 10 \\
\hline Locations $^{1}$ & 20 & 2 \\
\hline
\end{tabular}

1 The number of "center-to-limb" locations, or viewing geometries used to model each latitude region. 


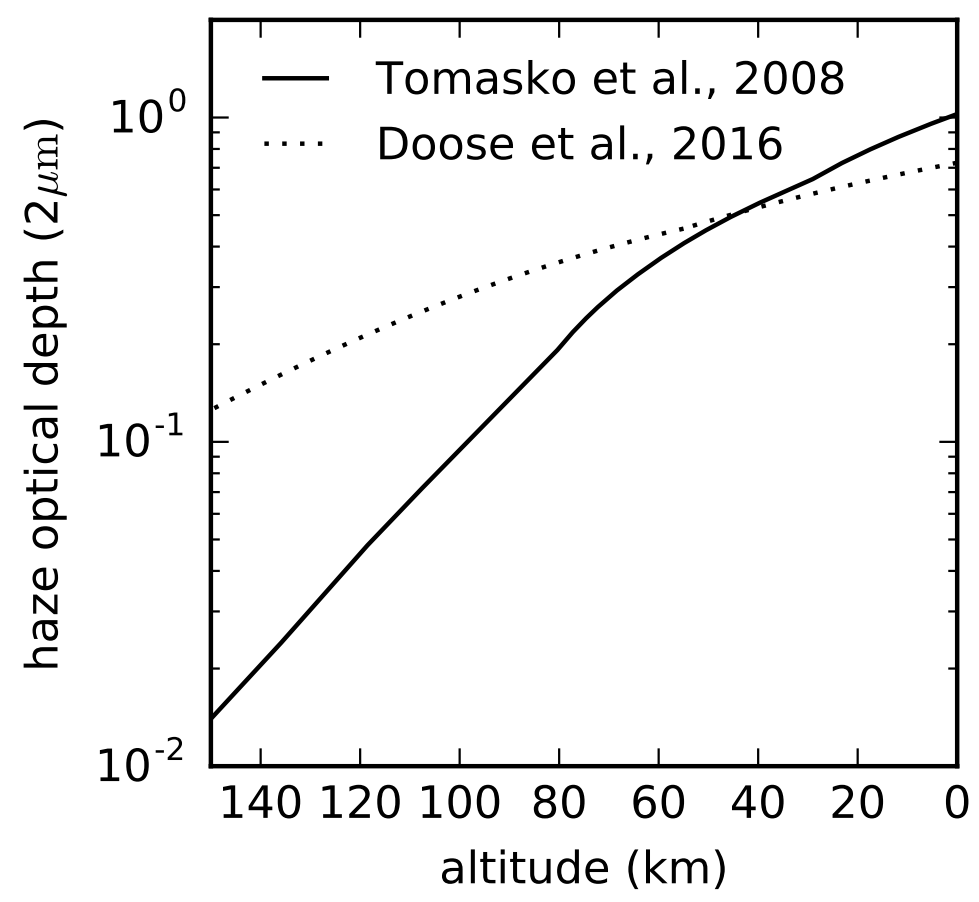

Figure 10: DISR haze models of the cumulative haze optical depth from the top of the atmosphere as reported by Tomasko et al. (2008), used as the nominal case for the radiative transfer models models here, and the recent revision by Doose et al. (2016), which is the nominal model used by Karkoschka (2016).

and $\tau_{H}=0.37$ from $80 \mathrm{~km}$ to the surface in the Doose et al. (2016) model DISR haze. The two haze scale factors, and their seasonal variation, that are reported by Karkoschka (2016) can be converted variation in $\tau_{H}$ at $2 \mu \mathrm{m}$. These curves are illustrated in Figure 11 (refer to the right axis of Figure 15 in Karkoschka (2016) for comparison).

In our nominal models, following Tomasko et al. (2008), we have $\tau_{H}=0.26$ below $20 \mathrm{~km}$ which we use to convert our scale factor $f_{H}$ to $\tau_{H}$ for comparison. Figure 10 shows that there is about $\tau_{H}=0.63$ above $20 \mathrm{~km}$ in the Tomasko et al. (2008) model. Using this scale factor would result in factors of a few to several larger variation in optical depth than in reported by Karkoschka (2016). This is likely due to the altitude sensitivity of our radiative transfer 
model. Although we vary the column of haze above $20 \mathrm{~km}$ in our model to determine $f_{S}$, the bulk of the spectral response in our model is from the upper-most layers of the model. The bottom level of the first layer of the model has a nominal optical depth of 0.11 in the Tomasko et al. (2008) model, which we used to scale our $f_{S}$ to $\tau_{H}$ for comparison with the results of Karkoschka (2016). Figure 11 shows a comparison of the optical depth variation at low and high altitudes for six latitude regions compared to the optical depth variation predicted by the seasonal model of Karkoschka (2016). While there is general agreement in the magnitude of change and some trends with latitude, there is significantly larger variation within individual latitude bands than predicted from the seasonal model.

Although significant decreases in stratospheric haze are consistent with spacecraft measurements, e.g., the dark band observed by Voyager (Smith et al., 1981), the decreases in $f_{S}$ near the poles are only faintly visible, if at all, in the images presented here that are sensitive to the stratosphere (e.g., on 2006-01-15 and 2010-01-29, in Figure 3). At wavelengths with significant methane absorption the atmosphere becomes optically thick for a column of haze scattering that is optically thin. For increasing airmass near the limb, the increased slant path through low density gas corresponds to larger scattering optical depths, and brighter observed flux. Although we exclude pixels close to the limb, at $\mu<0.68$, there may be effects of limb brightening that are exacerbated due to the observational point spread function (PSF).The proximity of the decrease in haze scattering opacity next to the significant limb brightening may decrease the contrast in the images. Artifacts in the data are also a concern and may obscure the features in the images sensitive to the upper haze layers. The mechanical lenslet mask for OSIRIS degraded in (re)positioning accuracy over time, causing scattered light from neighboring lenslets to contaminate the edge of the FOV, with the lenslet mask breaking completely in 2011. These lenslet masking artifacts near the edge of the OSIRIS FOV cause the vertical striping that is either faintly or, as in the case of observations on 2011-01-10, dramatically visible.

One of the primary concerns in interpreting the structure in upper haze variation seen in Figure 8 is possible artifacts in the retrieval of $f_{S}$ near the limb. Along with the arguments described above to support the validity of these features, we can also compare our results to similar analysis of the haze opacity variation using Cassini VIMS. Rannou et al. (2010) use a radiative transfer model with a correction for spherical geometry (SPDISORT; Mayer and Kylling, 2005) to interpret a full disk VIMS image ( $13^{\circ}$ phase angle), and 


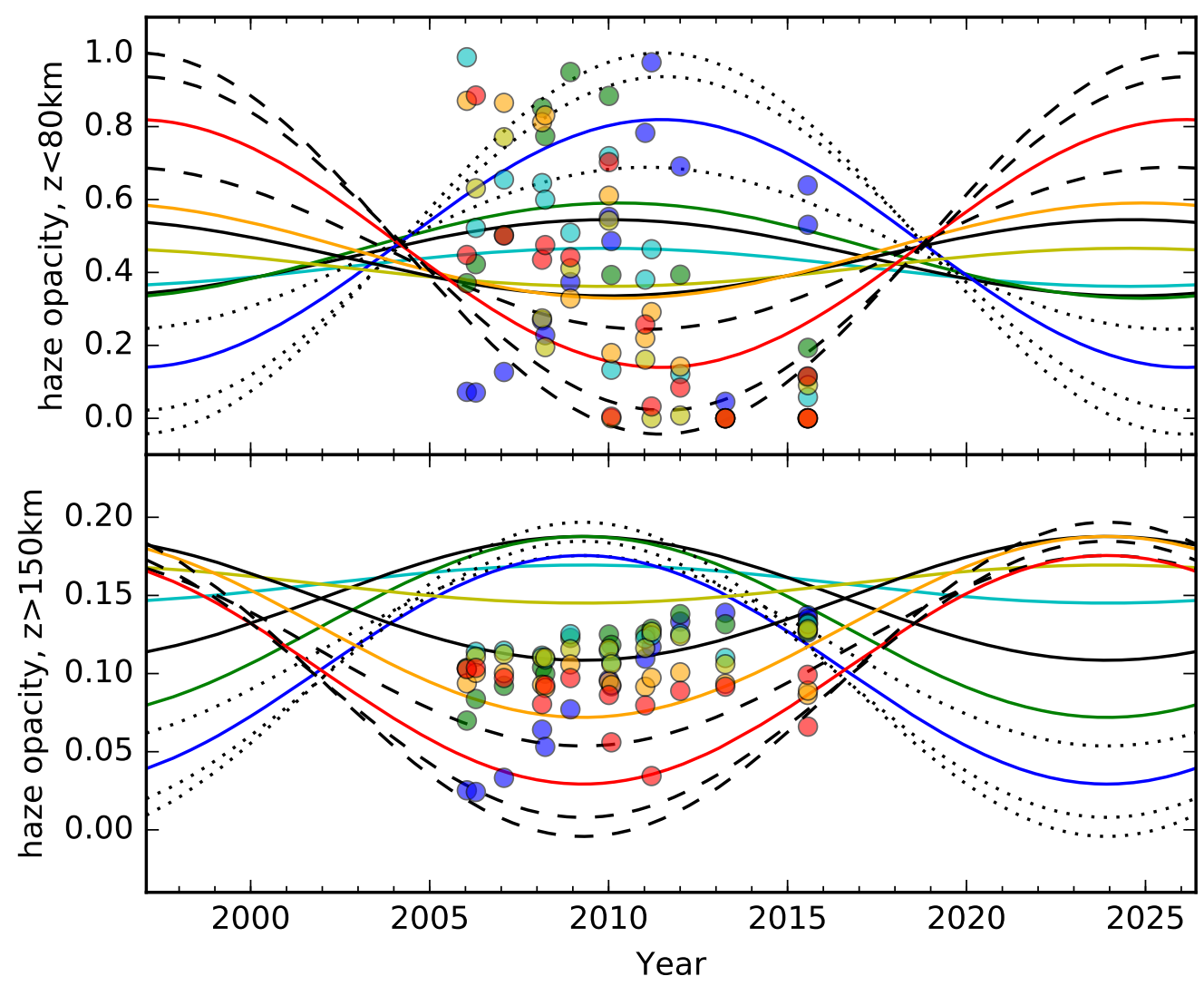

Figure 11: Seasonal variation in hazes opacity from the model of Karkoschka (2016) (curves, reproducing their line styles), with some latitudes highlighted in color for comparison; $45^{\circ} \mathrm{N}$ (blue), $25^{\circ} \mathrm{N}$ (green), $5^{\circ} \mathrm{N}$ (cyan), $5^{\circ} \mathrm{S}$ (yellow), $25^{\circ} \mathrm{S}$ (orange), $45^{\circ} \mathrm{S}$ (red). Datapoints retrieved form corresponding latitudes over the timespan of the observations are plotted in matching colors. 
find a linear increase in haze from $80^{\circ} \mathrm{S}$ to the Equator, where there is a sharp transition, and then uniform haze to $\sim 20^{\circ} \mathrm{N}$, and then a decrease in opacity to $60^{\circ} \mathrm{N}$ (their Figure 3). Their analysis of an image of the North polar region (at $113^{\circ}$ phase angle) from 2006-12-22 confirms the decrease in haze toward the North polar region. Our observations from 2006-04-17 and 2007-01-29 are closest in time; the former shows a discontinuity in the haze in the tropics (although not at the Equator as in 2004), while the latter has a corresponding decrease in haze from $20^{\circ} \mathrm{N}$ to the pole. The consistent interpretation between the different observations, made using different radiative transfer models, suggests that the poleward decreases in $f_{S}$ reported here (Figure 8) are real structure.

At $40^{\circ} \mathrm{S}$ we observe a decrease in $f_{S}$ from 2006 through 2011, after which this latitude is too close to the limb for accurate retrieval. Similarly, at $40^{\circ} \mathrm{N}$, we see an increase in haze from 2009 through 2013. At mid-latitudes, and in the tropics, there's no apparent trend with season. A transition near $20^{\circ} \mathrm{S}$ is observed in 2006 and 2008, but is not seen in 2007. In 2011, there a sharp drop in haze from the Equator southward through $20^{\circ} \mathrm{S}$, without a transition. Linear gradients in the absence of either significant haze decreases near the limb or discontinuities are only observed in 2013 and 2015. The lack of simple trends in $f_{S}$ with latitude and season haze suggests that a stochastic component of the haze variation could be important.

The haze in the lower atmosphere (Figure 9) is even more challenging to interpret in terms of a simple model of seasonal haze variation. In the lower atmosphere there are various bumps and wiggles atop possible linear meridional gradients in $f_{T}$. One of the interesting features in the retrievals of haze below $20 \mathrm{~km}$ is the decrease in $f_{T}$ between the $\sim 20^{\circ} \mathrm{S}$ and the Equator, on 2008-02-22, 2008-03-25, 2011-01-10, and 2011-03-15. On each of these dates, the sub-observer latitude (Table 1 and bottom right in each panel in Figure 9), is within $30^{\circ}$ of $125^{\circ} \mathrm{W}$, which is the bright region of Xanadu. The results here would suggest that there is a persistent hole in the haze below $20 \mathrm{~km}$ near Xanadu, which seems an unlikely interpretation of the observations. We've previously argued for an enhancement in methane opacity near Xanadu, with the interpretation of increased methane condensation (Ádámkovics et al., 2007, 2009). Here, since we assume a uniform methane opacity, we find that the parameter optimization for fitting the haze must decreased the haze to zero in order to fit the spectra. Decreasing the haze opacity below $20 \mathrm{~km}$ decreases the scattering and lowers $I / F$, resulting a spectral response that is similar to an increase in methane absorption. The 
spectra over Xanadu are systematically different than from other locations on Titan, and it seems that a spatially localized increase in the methane opacity is more likely than a localized decrease in haze scattering. For example, clouds are supposedly indications of (relatively) small spatial scale variations in methane. One the other hand, variations in the haze seem to occur on a much larger scale (with the exception of near the poles) and are generally (if not always) uniform along latitude bands. If an increase in methane opacity is indeed the correct, it still remains unclear whether or not methane is in the form of an additional absorber in the atmosphere, a coating on haze particles, or a layer on the surface. An additional consideration for the possibility of increased methane columns over Xanadu is the regional topography. Cassini RADAR SARtopo and altimetry has shown the region of Xanadu to be topographically low region (Lorenz et al., 2013). There is roughly $3 \mathrm{~km}$ amagat of $\mathrm{CH}_{4}$ in the nominal model (or a column of $\sim 10^{25} \mathrm{~cm}^{-2}$ ), which would be increased by $10 \%$ if there were an additional $\mathrm{km}$ of path-length appended to the bottom of the atmosphere. An increase in $\mathrm{CH}_{4}$ of this magnitude is not sufficient to account for the decrease in flux that is commensurate with the decrease is $f_{T}$ that we observe.

In the radiative transfer models presented here, methane opacities in spectral bands are implemented using correlated k-coefficients (Goody et al., 1989) rather than mean opacities per spectral bands. Methane line lists (e.g., HITRAN2012) go as short as $\sim 1.08 \mu \mathrm{m}$, and do not cover the spectral regions in the HST analysis, therefore mean opacities must be used for that work. However, averaging over $5 \AA$ in the near-IR corresponds to hundred of transitions that are not resolved. Within and individual spectral band, there will be regions of high and low methane opacity, which are sensitive to the surface and upper atmosphere. Averaging opacities to a mean value results in an intermediate opacity with sensitivity to an intermediate altitude range. Therefore, a mean opacity for a spectral band that includes strong methane opacity variation may have a larger uncertainty in the altitude sensitivity. On the other hand, the simplistic approximation of linear scaling of haze above and below a single altitude boundary likely outweighs the uncertainties due to how the gas opacities are implemented in the radiative transfer.

\section{Conclusions}

We summarize and make the following conclusions from this analysis: 
- Seasonal trends in the haze above and below $20 \mathrm{~km}$ on Titan are retrieved from 9.5 years of Keck OSIRIS observations in the K-band using a radiative transfer model and LM optimization.

- The Northward linear gradient in haze above $20 \mathrm{~km}$ is punctuated by significant seasonal decreases near the poles.

- The haze below $20 \mathrm{~km}$ is not well described by a simple seasonal model with stochastic variations playing a potentially significant role.

- Retrievals of the haze near Xanadu are consistent with the suggestion that the methane opacity is underestimated there.

The archive of Cassini VIMS observations over this period provide a tremendous resource for comparison and validation of the variation in haze. Extending the approach presented here to systematically model the hazes for a significant set of VIMS observations would be the next step in determining the relative importance of seasonal vs. stochastic variation in the hazes on Titan.

\section{Acknowledgements}

This work was supported by NSF award 1008788. We wish to thank Eliot F. Young for contributing the OSIRIS dataset from his observations on 201304-06. We are grateful for the thoughtful and constructive reviews by Robert West and Ralph Lorenz that have significantly improved the manuscript. The data presented herein were obtained at the W.M. Keck Observatory, which is operated as a scientific partnership among the California Institute of Technology, the University of California and the National Aeronautics and Space Administration. The Observatory was made possible by the generous financial support of the W.M. Keck Foundation. The authors wish to recognize and acknowledge the very significant cultural role and reverence that the summit of Mauna Kea has always had within the indigenous Hawaiian community. We are most fortunate to have the opportunity to conduct observations from this mountain.

\section{References}

Ádámkovics, M., Barnes, J. W., Hartung, M., de Pater, I., Aug. 2010. Observations of a stationary mid-latitude cloud system on Titan. Icarus 208, 868-877. 
Ádámkovics, M., de Pater, I., Hartung, M., Barnes, J. W., Nov. 2009. Evidence for condensed-phase methane enhancement over Xanadu on Titan. Planet. Space Sci. 57, 1586-1595.

Ádámkovics, M., de Pater, I., Hartung, M., Eisenhauer, F., Genzel, R., Griffith, C. A., Jun. 2006. Titan's bright spots: Multiband spectroscopic measurement of surface diversity and hazes. Journal of Geophysical Research (Planets) 111 (E7), E07S06.

Ádámkovics, M., de Pater, I., Roe, H. G., Gibbard, S. G., Griffith, C. A., Aug. 2004. Spatially-resolved spectroscopy at $1.6 \mu \mathrm{m}$ of Titan's atmosphere and surface. Geophys. Res. Lett. 31 (17), L17S05.

Ádámkovics, M., Mitchell, J. L., Hayes, A. G., Rojo, P. M., Corlies, P., Barnes, J. W., Ivanov, V. D., Brown, R. H., Baines, K. H., Buratti, B. J., Clark, R. N., Nicholson, P. D., Sotin, C., May 2016. Meridional variation in tropospheric methane on Titan observed with AO spectroscopy at Keck and VLT. Icarus 270, 376-388.

Ádámkovics, M., Wong, M. H., Laver, C., de Pater, I., Nov. 2007. Widespread Morning Drizzle on Titan. Science 318, 962-965.

Allen, M., Yung, Y. L., Pinto, J. P., Dec. 1980. Titan - Aerosol photochemistry and variations related to the sunspot cycle. ApJ 242, L125-L128.

Anderson, C. M., Young, E. F., Chanover, N. J., McKay, C. P., Apr. 2008. HST spectral imaging of Titan's haze and methane profile between 0.6 and $1 \mu \mathrm{m}$ during the 2000 opposition. Icarus 194, 721-745.

Barnes, J. W., Brown, R. H., Soderblom, L., Buratti, B. J., Sotin, C., Rodriguez, S., Le Mouèlic, S., Baines, K. H., Clark, R., Nicholson, P., Jan. 2007. Global-scale surface spectral variations on Titan seen from Cassini/VIMS. Icarus 186, 242-258.

Brown, M. E., Bouchez, A. H., Griffith, C. A., Dec. 2002. Direct detection of variable tropospheric clouds near Titan's south pole. Nature 420, 795-797.

Brown, R. H., Baines, K. H., Bellucci, G., Bibring, J.-P., Buratti, B. J., Capaccioni, F., Cerroni, P., Clark, R. N., Coradini, A., Cruikshank, D. P., Drossart, P., Formisano, V., Jaumann, R., Langevin, Y., Matson, D. L., McCord, T. B., Mennella, V., Miller, E., Nelson, R. M., Nicholson, P. D., 
Sicardy, B., Sotin, C., Dec. 2004. The Cassini Visual And Infrared Mapping Spectrometer (Vims) Investigation. Space Science Reviews 115, 111-168.

Coustenis, A., Bezard, B., May 1995. Titan's atmosphere from Voyager infrared observations. 4: Latitudinal variations of temperature and composition. Icarus 115, 126-140.

de Kok, R., Irwin, P. G. J., Teanby, N. A., Vinatier, S., Tosi, F., Negrão, A., Osprey, S., Adriani, A., Moriconi, M. L., Coradini, A., May 2010. A tropical haze band in Titan's stratosphere. Icarus 207, 485-490.

de Pater, I., Ádámkovics, M., Bouchez, A. H., Brown, M. E., Gibbard, S. G., Marchis, F., Roe, H. G., Schaller, E. L., Young, E., Jun. 2006. Titan imagery with Keck adaptive optics during and after probe entry. Journal of Geophysical Research (Planets) 111, 7-+.

Doose, L. R., Karkoschka, E., Tomasko, M. G., Anderson, C. M., May 2016. Vertical structure and optical properties of Titan's aerosols from radiance measurements made inside and outside the atmosphere. Icarus 270, 355375 .

Gibbard, S. G., de Pater, I., Macintosh, B. A., Roe, H. G., Max, C. E., Young, E. F., McKay, C. P., Jul. 2004. Titan's $2 \mu \mathrm{m}$ surface albedo and haze optical depth in 1996-2004. Geophys. Res. Lett. 31, 17-+.

Gibbard, S. G., Macintosh, B., Gavel, D., Max, C. E., de Pater, I., Ghez, A. M., Young, E. F., McKay, C. P., Jun. 1999. Titan: High-Resolution Speckle Images from the Keck Telescope. Icarus 139, 189-201.

Goody, R., West, R., Chen, L., Crisp, D., Dec. 1989. The correlated$\mathrm{k}$ method for radiation calculations in nonhomogeneous atmospheres. J. Quant. Spec. Radiat. Transf. 42, 539-550.

Griffith, C. A., Penteado, P., Rodriguez, S., LeMouélic, S., Baines, K. H., Buratti, B., Clark, R., Nicholson, P., Jaumann, R., Sotin, C., Sep. 2009. Characterization of Clouds in Titan's Tropical Atmosphere. ApJ 702, L105L109.

Hutzell, W. T., McKay, C. P., Toon, O. B., Sep. 1993. Effects of TimeVarying Haze Production on Titan's Geometric Albedo. Icarus 105, 162174. 
Hutzell, W. T., McKay, C. P., Toon, O. B., Hourdin, F., Jan. 1996. Simulations of Titan's Brightness by a Two-Dimensional Haze Model. Icarus 119, $112-129$.

Iserlohe, C., Krabbe, A., Larkin, J. E., Barczys, M., McElwain, M. W., Quirrenbach, A., Weiss, J., Wright, S. A., Aug. 2013. Near-infrared imaging spectroscopy of the inner few arcseconds of NGC 4151 with OSIRIS at Keck. A\&A 556, A136.

Karkoschka, E., May 2016. Seasonal variation of Titan's haze at low and high altitudes from HST-STIS spectroscopy. Icarus 270, 339-354.

Karkoschka, E., Lorenz, R. D., Feb. 1997. Latitudinal Variation of Aerosol Sizes Inferred from Titan's Shadow. Icarus 125, 369-379.

Khare, B. N., Sagan, C., Nov. 1973. Red Clouds in Reducing Atmospheres. Icarus 20, 311-321.

Krasnopolsky, V. A., May 2009. A photochemical model of Titan's atmosphere and ionosphere. Icarus 201, 226-256.

Larkin, J., Barczys, M., Krabbe, A., Adkins, S., Aliado, T., Amico, P., Brims, G., Campbell, R., Canfield, J., Gasaway, T., Honey, A., Iserlohe, C., Johnson, C., Kress, E., LaFreniere, D., Magnone, K., Magnone, N., McElwain, M., Moon, J., Quirrenbach, A., Skulason, G., Song, I., Spencer, M., Weiss, J., Wright, S., Jun. 2006. OSIRIS: A diffraction limited integral field spectrograph for Keck. New A Rev. 50, 362-364.

Larson, E. J. L., Toon, O. B., West, R. A., Friedson, A. J., Jul. 2015. Microphysical modeling of Titan's detached haze layer in a 3D GCM. Icarus $254,122-134$.

Laver, C., de Pater, I., Marchis, F., Nov. 2007. Tvashtar awakening detected in April 2006 with OSIRIS at the W.M. Keck Observatory. Icarus 191, 749-754.

Lavvas, P., Griffith, C. A., Yelle, R. V., Oct. 2011. Condensation in Titan's atmosphere at the Huygens landing site. Icarus 215, 732-750.

Lavvas, P. P., Coustenis, A., Vardavas, I. M., Jan. 2008a. Coupling photochemistry with haze formation in Titan's atmosphere, Part I: Model description. Planet. Space Sci. 56, 27-66. 
Lavvas, P. P., Coustenis, A., Vardavas, I. M., Jan. 2008b. Coupling photochemistry with haze formation in Titan's atmosphere, Part II: Results and validation with Cassini/Huygens data. Planet. Space Sci. 56, 67-99.

Lorenz, R. D., Brown, M. E., Flasar, F. M., 2010. Seasonal Change on Titan. p. 353 .

Lorenz, R. D., Lemmon, M. T., Smith, P. H., Lockwood, G. W., Dec. 1999. Seasonal Change on Titan Observed with the Hubble Space Telescope WFPC-2. Icarus 142, 391-401.

Lorenz, R. D., Smith, P. H., Lemmon, M. T., May 2004. Seasonal change in Titan's haze 1992-2002 from Hubble Space Telescope observations. Geophys. Res. Lett. 31, 10702-+.

Lorenz, R. D., Stiles, B. W., Aharonson, O., Lucas, A., Hayes, A. G., Kirk, R. L., Zebker, H. A., Turtle, E. P., Neish, C. D., Stofan, E. R., Barnes, J. W., Jul. 2013. A global topographic map of Titan. Icarus 225, 367-377.

Lorenz, R. D., Young, E. F., Lemmon, M. T., Dec. 2001. Titan's Smile and Collar: HST Observations of Seasonal Change 1994-2000. Geophys. Res. Lett. 28, 4453-4456.

Mayer, B., Kylling, A., Jul. 2005. Technical note: The libRadtran software package for radiative transfer calculations - description and examples of use. Atmospheric Chemistry \& Physics 5, 1855-1877.

McKay, C. P., Coustenis, A., Samuelson, R. E., Lemmon, M. T., Lorenz, R. D., Cabane, M., Rannou, P., Drossart, P., Jan. 2001. Physical properties of the organic aerosols and clouds on Titan. Planet. Space. Sci 49, 79-99.

McKay, C. P., Pollack, J. B., Courtin, R., Jul. 1989. The thermal structure of Titan's atmosphere. Icarus 80, 23-53.

Meier, R., Smith, B. A., Owen, T. C., Terrile, R. J., Jun. 2000. The surface of Titan from NICMOS observations with the Hubble Space Telescope. Icarus 145, 462-473.

Mishchenko, M. I., Dlugach, J. M., Yurkin, M. A., Bi, L., Cairns, B., Liu, L., Panetta, R. L., Travis, L. D., Yang, P., Zakharova, N. T., May 2016. Firstprinciples modeling of electromagnetic scattering by discrete and discretely heterogeneous random media. Phys. Rep. 632, 1-75. 
Penteado, P. F., Griffith, C. A., Tomasko, M. G., Engel, S., See, C., Doose, L., Baines, K. H., Brown, R. H., Buratti, B. J., Clark, R., Nicholson, P., Sotin, C., Mar. 2010. Latitudinal variations in Titan's methane and haze from Cassini VIMS observations. Icarus 206, 352-365.

Podolak, M., Noy, N., Bar-Nun, A., Nov. 1979. Photochemical aerosols in Titan's atmosphere. Icarus 40, 193-198.

Rages, K., Pollack, J. B., Jan. 1980. Titan aerosols - Optical properties and vertical distribution. Icarus 41, 119-130.

Rages, K., Pollack, J. B., Jul. 1983. Vertical distribution of scattering hazes in Titan's upper atmosphere. Icarus 55, 50-62.

Rages, K., Pollack, J. B., Smith, P. H., Nov. 1983. Size estimates of Titan's aerosols based on Voyager high-phase-angle images. J. Geophys. Res. 88, 8721-8728.

Rannou, P., Cours, T., Le Mouélic, S., Rodriguez, S., Sotin, C., Drossart, P., Brown, R., Aug. 2010. Titan haze distribution and optical properties retrieved from recent observations. Icarus 208, 850-867.

Rannou, P., Ferrari, C., Rages, K., Roos-Serote, M., Cabane, M., Sep. 2000. Characterization of Aerosols in the Detached Haze Layer of Titan. Icarus 147, 267-281.

Rannou, P., Hourdin, F., McKay, C. P., Aug. 2002. A wind origin for Titan's haze structure. Nature 418, 853-856.

Rannou, P., Hourdin, F., McKay, C. P., Luz, D., Aug. 2004. A coupled dynamics-microphysics model of Titan's atmosphere. Icarus 170, 443-462.

Rannou, P., Le Mouélic, S., Sotin, C., Brown, R. H., Mar. 2012. Cloud and Haze in the Winter Polar Region of Titan Observed with Visual and Infrared Mapping Spectrometer on Board Cassini. ApJ 748, 4.

Roe, H. G., de Pater, I., Macintosh, B. A., Gibbard, S. G., Max, C. E., McKay, C. P., May 2002. NOTE: Titan's Atmosphere in Late Southern Spring Observed with Adaptive Optics on the W. M. Keck II 10-Meter Telescope. Icarus 157, 254-258. 
Rothman, L. S., Gordon, I. E., Babikov, Y., Barbe, A., Chris Benner, D., Bernath, P. F., Birk, M., Bizzocchi, L., Boudon, V., Brown, L. R., Campargue, A., Chance, K., Cohen, E. A., Coudert, L. H., Devi, V. M., Drouin, B. J., Fayt, A., Flaud, J.-M., Gamache, R. R., Harrison, J. J., Hartmann, J.-M., Hill, C., Hodges, J. T., Jacquemart, D., Jolly, A., Lamouroux, J., Le Roy, R. J., Li, G., Long, D. A., Lyulin, O. M., Mackie, C. J., Massie, S. T., Mikhailenko, S., Müller, H. S. P., Naumenko, O. V., Nikitin, A. V., Orphal, J., Perevalov, V., Perrin, A., Polovtseva, E. R., Richard, C., Smith, M. A. H., Starikova, E., Sung, K., Tashkun, S., Tennyson, J., Toon, G. C., Tyuterev, V. G., Wagner, G., Nov. 2013. The HITRAN2012 molecular spectroscopic database. J. Quant. Spec. Radiat. Transf. 130, 4-50.

Smith, B. A., Soderblom, L., Batson, R. M., Bridges, P. M., Inge, J. L., Masursky, H., Shoemaker, E., Beebe, R. F., Boyce, J., Briggs, G., Bunker, A., Collins, S. A., Hansen, C., Johnson, T. V., Mitchell, J. L., Terrile, R. J., Cook, A. F., Cuzzi, J. N., Pollack, J. B., Danielson, G. E., Ingersoll, A. P., Davies, M. E., Hunt, G. E., Morrison, D., Owen, T., Sagan, C., Veverka, J., Strom, R., Suomi, V. E., Jan. 1982. A new look at the Saturn system - The Voyager 2 images. Science 215, 504-537.

Smith, B. A., Soderblom, L., Beebe, R. F., Boyce, J. M., Briggs, G., Bunker, A., Collins, S. A., Hansen, C., Johnson, T. V., Mitchell, J. L., Terrile, R. J., Carr, M. H., Cook, A. F., Cuzzi, J. N., Pollack, J. B., Danielson, G. E., Ingersoll, A. P., Davies, M. E., Hunt, G. E., Masursky, H., Shoemaker, E. M., Morrison, D., Owen, T., Sagan, C., Veverka, J., Strom, R., Suomi, V. E., Apr. 1981. Encounter with Saturn - Voyager 1 imaging science results. Science 212, 163-191.

Stamnes, K., Tsay, S.-C., Jayaweera, K., Wiscombe, W., 1988. Numerically stable algorithm for discrete-ordinate-method radiative transfer in multiple scattering and emitting layered media. Applied Optics 27, 2502-2509.

Strobel, D. F., Apr. 1974. The Photochemistry of Hydrocarbons in the Atmosphere of Titan. Icarus 21, 466-470.

Tomasko, M. G., Archinal, B., Becker, T., Bézard, B., Bushroe, M., Combes, M., Cook, D., Coustenis, A., de Bergh, C., Dafoe, L. E., Doose, L., Douté, S., Eibl, A., Engel, S., Gliem, F., Grieger, B., Holso, K., HowingtonKraus, E., Karkoschka, E., Keller, H. U., Kirk, R., Kramm, R., Küppers, 
M., Lanagan, P., Lellouch, E., Lemmon, M., Lunine, J., McFarlane, E., Moores, J., Prout, G. M., Rizk, B., Rosiek, M., Rueffer, P., Schröder, S. E., Schmitt, B., See, C., Smith, P., Soderblom, L., Thomas, N., West, R., Dec. 2005. Rain, winds and haze during the Huygens probe's descent to Titan's surface. Nature 438, 765-778.

Tomasko, M. G., Doose, L., Engel, S., Dafoe, L. E., West, R., Lemmon, M., Karkoschka, E., See, C., Apr. 2008. A model of Titan's aerosols based on measurements made inside the atmosphere. Planet. Space Sci. 56, 669-707.

Toon, O. B., McKay, C. P., Griffith, C. A., Turco, R. P., Jan. 1992. A physical model of Titan's aerosols. Icarus 95, 24-53.

West, R. A., Balloch, J., Dumont, P., Lavvas, P., Lorenz, R., Rannou, P., Ray, T., Turtle, E. P., Mar. 2011. The evolution of Titan's detached haze layer near equinox in 2009. Geophys. Res. Lett. 38, 6204.

Willacy, K., Allen, M., Yung, Y., Oct. 2016. A New Astrobiological Model of the Atmosphere of Titan. ApJ 829, 79.

Wilson, E. H., Atreya, S. K., Jun. 2004. Current state of modeling the photochemistry of Titan's mutually dependent atmosphere and ionosphere. Journal of Geophysical Research (Planets) 109, E06002.

Wizinowich, P., Acton, D. S., Shelton, C., Stomski, P., Gathright, J., Ho, K., Lupton, W., Tsubota, K., Lai, O., Max, C., Brase, J., An, J., Avicola, K., Olivier, S., Gavel, D., Macintosh, B., Ghez, A., Larkin, J., Mar. 2000. First Light Adaptive Optics Images from the Keck II Telescope: A New Era of High Angular Resolution Imagery. Pub. Astron. Sci. Pac. 112, 315-319.

Young, E. F., Rannou, P., McKay, C. P., Griffith, C. A., Noll, K., Jun. 2002. A Three-dimensional Map of Titan's Tropospheric Haze Distribution Based on Hubble Space Telescope Imaging. Astron. J. 123, 3473-3486.

Yung, Y. L., Allen, M., Pinto, J. P., Jul. 1984. Photochemistry of the atmosphere of Titan - Comparison between model and observations. Astrophys. J. Supp. Ser. 55, 465-506. 